\title{
THE ROLE OF COUNTABLE DIMENSIONALITY IN THE THEORY OF CELL-LIKE RELATIONS
}

\author{
BY \\ FREDRIC D. ANCEL ${ }^{1}$
}

\begin{abstract}
Consider only metrizable spaces. The notion of a slice-trivial relation is introduced, and Theorem 3.2 is proved. This theorem sets forth sufficient conditions for a continuous relation with compact $U V^{\infty}$ point images to be slice-trivial. Theorem 4.5 posits a number of necessary and sufficient conditions for a map to be a hereditary shape equivalence. Several applications of these two theorems are made, including the following.

THEOREM 5.1. A cell like map $f: X \rightarrow Y$ is a hereditary shape equivalence if there is a sequence $\left\{K_{n}\right\}$ of closed subsets of $Y$ such that

(1) $Y-\bigcup_{n=1}^{\infty} K_{n}$ is countable dimensional, and

(2) $f \mid f^{-1}\left(K_{n}\right): f^{-1}\left(K_{n}\right) \rightarrow K_{n}$ is a hereditary shape equivalence for each $n \geq 1$.

THEOREM 5.9. If $f: X \rightarrow Y$ is a proper onto map whose point inverses are $U V^{\infty}$ sets, then $Y$ is an absolute neighborhood extensor for the class of countable dimensional spaces. Furthermore, if $Y$ is countable dimensional, then $Y$ is an absolute neighborhood retract.
\end{abstract}

Theorem 5.9 is of particular interest when specialized to the identity map of a locally contractible space.

1. Introduction. This is an investigation of continuous relations whose point images are compact cell-like or $U V^{\infty}$ sets. Central to this study is the concept of slice-triviality, defined in $\S 2$. Main Theorem 3.2 gives a sufficient condition for a continuous relation with compact $U V^{\infty}$ point images to be slice-trivial. This condition requires that a certain critical subset of the domain of the relation be countable dimensional. Hence the title of the paper.

We are motivated to consider continuous relations with cell-like point images in order to attain new insight into the study of cell-like maps. The inverse of a celllike map is, in fact, a continuous relation with cell-like point images. However, the power of the techniques developed here does not arise from a purely formal switch from a map to its inverse. It comes from an emphasis on the relation as an object to be manipulated. In other words, we modify a function or relation by directly manipulating its graph rather than performing adjustments in its domain or range. The notion of slice-triviality ( $(2)$ typifies the approach to relations taken here.

I initially undertook this study not to extend known results on cell-like maps, but merely to understand them in relation-theoretic terms. I was led to extensions and unifications of the known theorems. This can be regarded as evidence that the relation-theoretic point of view provides stronger control of cell-like maps and relations than was formerly available.

Received by the editors March 22, 1982 and, in revised form, August 29, 1983.

1980 Mathematics Subject Classification. Primary 54C55, 54C56, 54F45; Secondary 54C60.

${ }^{1}$ Partially supported by N.S.F. Grant No. MCS 8103420. 
The perception that the study of cell-like maps would be enriched by a welldeveloped theory of cell-like relations is due to J. W. Cannon. Cannon laid the foundations of this theory in $[\mathbf{C 1}]$. Cell-like relations have had several interesting applications $[\mathbf{A}, \mathbf{A C}, \mathbf{C 1}-\mathbf{C 4}, \mathbf{E v}]$.

We now describe the contents of this paper.

The remainder of this section is devoted to a description of the current state of understanding of cell-like maps and of the place occupied by the results of this paper. This discussion is accompanied by the definitions of certain basic concepts necessary to understand it and the rest of the paper.

$\S 2$ states the definition of slice-triviality (a central concept in this paper) and explores some of its ramifications. $\S 3$ defines " $U V V^{\infty}$ relation" and then gives a proof of Main Theorem 3.2.

$\S 4$ is devoted to a comparison of the concepts of slice-triviality, fine homotopy equivalence and hereditary shape equivalence. (Definitions of the latter two terms are given therein.) Theorem 4.5 summarizes the results of $\S 4$. Following Theorem 4.5 is a discussion of several open problems suggested by the theorems in this section, including comments on the notion of uniform local contractibility.

$\S 5$ provides three applications of Main Theorem 3.2 together with some corollaries. The first application, Theorem 5.1, gives conditions on a cell-like map which are sufficient to make the map a hereditary shape equivalence; this theorem generalizes results of Kozlowski [K2] . The second application, Theorem 5.9, derives certain conclusions about the image space of a $U V^{\infty}$ map which are particularly interesting when specialized to the identity map of a locally contractible space. The third application, to be found in Theorem 5.14, is a new proof that a cell-like map is a fine homotopy equivalence if its domain and range are absolute neighborhood retracts (ANR's); this was first established by Haver in $[\mathbf{H 1}]$ with a separability hypothesis that we avoid.

Following $\S 5$ are three appendices. Appendix $\mathrm{A}$ is a primer on continuous relations for the reader's convenience; its most significant result is the Enlargement Lemma A.8. Appendix B is a summary of those properties of polyhedra with the metric topology that are relevant to the study of ANR's; it appears here because the standard references tend to omit this material in favor of results on polyhedra with the Whitehead topology. In Appendix C, Main Theorem 3.2 and its corollaries are strengthened by replacing the hypothesis of countable dimensionality by property C. Property C, which was first introduced by W. E. Haver in $[\mathbf{H 2}]$, captures an essential attribute of countable dimensionality. Indeed, property $\mathrm{C}$ is the precise aspect of countable dimensionality required to prove Main Theorem 3.2. All countable dimensional spaces have property $\mathrm{C}$. However, as was recently revealed by the striking example of $\mathrm{R}$. Pol $[\mathbf{P}]$, property $\mathrm{C}$ encompasses a demonstrably larger class of spaces than does countable dimensionality. The impact of Pol's example on the results of this paper is relegated to Appendix $\mathrm{C}$, only because the body of this paper was written before the significance of Pol's example was recognized.

We now recall the definitions of the basic terms of the subject.

First, for the record, we give convenient definitions of zero-, finite and countable dimensionality (see $[\mathbf{N}$, p. 9, Theorem II.4 on p. 19, Theorem II.6 on p. 22, and p. $162]$ ). Let $X$ be a metrizable space. $X$ is zero-dimensional if every open cover of $X$ is refined by an open cover whose elements are disjoint. $X$ is finite dimensional 
if $X$ is the union of a finite number of zero-dimensional subspaces. If $X$ is finite dimensional, the dimension of $X$, denoted $\operatorname{dim} X$, is the least integer $n \geq 0$ such that $X$ is the union of $n+1$ zero-dimensional subspaces. $X$ is countable dimensional if $X$ is the union of a countable number of zero-dimensional subspaces. It may help to keep the following two examples in mind. The Hilbert cube $[0,1] \times[0,1] \times[0,1] \times \cdots$ is not countable dimensional [N, Theorem VI.1.B, p. 164]). However, the subset $\bigcup_{n=1}^{\infty}[0,1]^{n} \times\{0\} \times\{0\} \times\{0\} \times \cdots$ of the Hilbert cube is countable dimensional.

A space $X$ is an absolute neighborhood retract (ANR) if $X$ is metrizable and if for every closed embedding $e: X \rightarrow Y$ into a metrizable space $Y$, there is a neighborhood $L$ of $e(X)$ in $Y$ and a map $r: L \rightarrow X$ such that $r \circ e=1 \mid X$.

Let $\mathfrak{C}$ be a class of metrizable spaces. A space $Y$ is an absolute neighborhood extensor (ANE) for $\mathfrak{C}$ if $Y$ is metrizable and satisfies the following condition. If $A$ is a closed subset of a space $X \in \mathfrak{C}$, then any map $f: A \rightarrow Y$ extends to a map $g: L \rightarrow Y$ where $L$ is a neighborhood of $A$ in $X$.

It is well known that a metrizable space is an ANR if and only if it is an ANE for the class of all metrizable spaces (see Theorems III.3.1 and III.3.2, pp. 83-86 of $[\mathbf{H u}])$.

Let $V$ be a subset of a space $U$. A contraction of $V$ in $U$ is a homotopy $\phi: V \times$ $[0,1] \rightarrow U$ such that $\phi_{0}=1 \mid V$ and $\phi_{1}(V)$ is a point. If there is a contraction of $V$ in $U$, we say $V$ contracts in $U$ or $V$ is contractible in $U$.

A subset $A$ of a space $X$ is a $U V^{\infty}$ subset of $X$ if each neighborhood $U$ of $A$ in $X$ contains a neighborhood $V$ of $A$ in $X$ such that $V$ contracts in $U$.

A space $X$ is cell-like if it is a compact metrizable space which satisfies any one of the following three equivalent conditions.

(1) Any map from $X$ to an ANR is homotopic to a constant map.

(2) There is an embedding $e: X \rightarrow Y$ into a metrizable space $Y$ such that $e(X)$ contracts in each of its neighborhoods in $Y$.

(3) For every embedding $e: X \rightarrow Y$ into an ANR $Y, e(X)$ is a $U V^{\infty}$ subset of $Y$.

The equivalence of these conditions was first recorded in [L1]. The proof follows easily from basic properties of ANR's.

A more geometric characterization of cell-likeness can be given. A compact metrizable space $X$ is cell-like if and only if there is an embedding $e: X \rightarrow \mathbf{Q}$, where $\mathbf{Q}$ is the Hilbert cube, such that $\mathbf{Q}-e(X)$ is homeomorphic to $\mathbf{Q}-\{q\}$ for some $q \in \mathbf{Q}$ [Ch, Theorem 2.5.2, p. 40]. Moreover, if $X$ is finite dimensional, then $\mathbf{Q}$ can be replaced by a Euclidean space of sufficiently high dimension in the preceding equivalence $[\mathbf{M}]$.

There is also a shape-theoretic characterization of cell-likeness. A compact metrizable space is cell-like if and only if it has the shape of a point.

Cell-likeness is a topological property. However, $U V^{\infty}$ is a property of the embedding of the given space in the ambient space. Indeed, a point is always cell-like; but it may fail to be a $U V^{\infty}$ subset of its ambient space if the ambient space is not locally contractible. This situation occurs at the point 0 in the subspace $\left\{0, \frac{1}{2}, \frac{1}{3}, \frac{1}{4}, \ldots\right\}$ of the real line.

In addition to finite dimensional cells and the Hilbert cube, a list of topologically prominent cell-like spaces must include the following:

(1) the topologist's sine wave [SS, counterexample 117, pp. 137-138],

(2) the pseudo-arc [SS, counterexample 130, pp. 147-148]), 
(3) the dunce hat $[\mathbf{Z}]$,

(4) the Knaster continuum [Ku, pp. 205-206]), and its three-dimensional manifestation,

(5) the Whitehead continuum (the set $X$ defined on p. 279 of $[\mathbf{W}]$ ).

We are using the term map to denote a continuous function. Let $f: X \rightarrow Y$ be a map. $f$ is a closed map if $f(A)$ is a closed subset of $Y$ whenever $A$ is a closed subset of $X$. Any set of the form $f^{-1}(y)$, where $y \in Y$, is called a point inverse of $f . f$ is a proper map if it is a closed map and if each of its points inverses is compact. If $Y$ is a Hausdorff space which either satisfies the first axiom of countability or is locally compact, then the propriety of $f$ is equivalent to the condition that $f^{-1} A$ be compact whenever $A$ is a compact subset of $Y$.

A map $f: X \rightarrow Y$ is a $U V^{\infty}$ map if $f$ is a proper onto map and each of its point inverses is a $U V^{\infty}$ subset of $X$. A map $f: X \rightarrow Y$ is a cell-like map if $f$ is a proper onto map and each of its point inverses is cell-like.

A decomposition $G$ of a space $X$ is called a cell-like (upper semicontinuous) decomposition of $X$ if the quotient map $X \rightarrow X / G$ is a cell-like map.

Let $G$ be a cell-like decomposition of a finite dimensional manifold $M$. In general $M / G$ is not a manifold (unless $\operatorname{dim} M \leq 2$ ). However, in all known cases $(M / G) \times \mathbf{R}$ is homeomorphic $M \times \mathbf{R}$. Deciding whether this is always the case is one of the outstanding unresolved problems about cell-like decompositions.

Cell-like decompositions of finite dimensional manifolds have played a central role in several important recent advances in topology. Foremost among these are the solution of the double suspension problem by R. D. Edwards $[\mathbf{E}]$ and J. W. Cannon [C4], H. Torunczyk's characterization of Hilbert cube manifolds [To], and M. Freedman's recent resolution of the four-dimensional Poincaré conjecture $[\mathbf{F}]$.

Let $G$ be a cell-like decomposition of a finite dimensional manifold $M$. Because the quotient map $q: M \rightarrow M / G$ has point inverses of trivial shape, it is natural to expect $q$ to be a homotopy equivalence. In all known cases, $q$ is a homotopy equivalence; and deciding whether this is always the case is perhaps the principal unresolved problem about cell-like decompositions.

Most of the significant geometric arguments about cell-like decompositions of finite dimensional manifolds depend crucially on the existence of homotopy inverses to the quotient maps. For this reason, the question of when a cell-like map is a homotopy equivalence is studied in its own right. This question motivates much of the work in the present paper.

Consider a cell-like map whose domain is a manifold or, more generally, an ANR. The potential exists for the map to fail to be a homotopy equivalence. Such pathology is exhibited by the Taylor example, which is discussed below. However, in the absence of such pathology, we can expect the map to be a strong sort of homotopy equivalence called a fine homotopy equivalence (defined at the beginning of $\S 4$ ). The following by-now-classical theorem reveals the foundation of our knowledge about the relation between cell-like maps and fine homotopy equivalences.

1.1. THEOREM. Let $f: X \rightarrow Y$ be a cell-like map from an ANR $X$ to a metrizable space $Y$. Then the following two conditions are equivalent.

(1) $f$ is a fine homotopy equivalence. 
(2) $Y$ is an $A N R$.

Furthermore, if $\operatorname{dim} X<\infty$, then the following two conditions can be added to this list.

(3) $Y$ is finite dimensional.

(4) $\operatorname{dim} Y \leq \operatorname{dim} X$.

The history of this theorem begins with Smale's homotopy version $[\mathbf{S m}]$ of the Vietoris homology mapping theorem [Sp, Theorem 15, p. 344]). Proofs restricted to the case in which $X$ and $Y$ are finite dimensional are presented in $[\mathbf{A P}, \mathbf{K 1}$ and L2]. A proof appears in [H1] of the equivalence of (1) and (2) with no restriction on the dimension of $X$ or $Y$, but under the hypothesis that $X$ and $Y$ are separable. Theorem 1.1 as stated here follows from the work in [K2]. However, since $[\mathbf{K 2}]$ does not deal directly with the concept of fine homotopy equivalence, some translation is necessary to obtain Theorem 1.1 from the results of $[\mathbf{K 2}]$; the connections needed for this translation are provided (for example) by the results in $\S 4$ of this paper.

All but one of the implications of Theorem 1.1 can be deduced from results in this paper. The equivalence of conditions (1) and (2) follows from Theorem 5.14 and Corollary 4.8. Also, the implication from condition (3) to condition (1) is a consequence of Corollary 5.3.

The equivalence of conditions (1) and (2) in Theorem 1.1 does not hold by default. The Taylor example, alluded to earlier, leads to a cell-like map $\tau_{+}$whose domain is the Hilbert cube and which is not a fine homotopy equivalence. Consequently its range cannot be an ANR. (The map constructed by Taylor in [T] is a cell-like map $\tau$ whose domain is a compact metrizable space, whose range is the Hilbert cube, and which is not a shape equivalence. The map $\tau_{+}$described above is, in the language of $\S 4$ of this paper, a trivial extension of the map $\tau$ to a map whose domain is the Hilbert cube.)

The principal unresolved problem about cell-like decompositions mentioned above can be rephrased as follows. Is there an example like the Taylor example with a finite dimensional domain? In light of Theorem 1.1, this question takes the following form.

1.2 The principal unresolved problem about cell-like decompositions. Is there a cell-like map $f: X \rightarrow Y$ from a finite dimensional ANR $X$ to a metrizable space $Y$ such that any one of the following equivalent conditions holds?

(1) $f$ is not a fine homotopy equivalence.

(2) $Y$ is not an ANR.

(3) $Y$ is infinite dimensional.

(4) $\operatorname{dim} Y>\operatorname{dim} X$.

Because of condition (4), this problem is commonly called the dimension raising cell-like map question. One consequence of Corollary 5.3 of this paper, as well as of the work in [K2], is that if there is a cell-like map $f: X \rightarrow Y$ from a finite dimensional ANR $X$ to a metrizable space $Y$ with $\operatorname{dim} Y>\operatorname{dim} X$, then $Y$ cannot even be countable dimensional.

Let $f: X \rightarrow Y$ be a cell-like map from an ANR $X$ to a metrizable space $Y$. Theorem 1.1 and the Taylor example naturally delineate the problem of discovering sufficient conditions for $f$ to be a fine homotopy equivalence. G. Kozlowski investigated this problem and recorded many of his results in [K2]. Theorem 5.1 of this paper together with its corollaries include and extend a number of theorems 
in [K2]. For instance, Corollary 5.2 implies that $f$ is a fine homotopy equivalence if the singular set of $f$,

$$
\left\{y \in Y: f^{-1}(y) \text { contains more than one point }\right\},
$$

lies in a countable dimensional $G_{\delta}$ subset of $Y$.

Recent work of R. J. Daverman and J. J. Walsh in [DW] shows that the hypotheses of Corollary 5.2 are close to being minimal. They modify the Taylor example to provide a cell-like map whose domain is the Hilbert cube, which is not a fine homotopy equivalence, and whose singular set is countable dimensional. Hence this singular set is a countable dimensional subset of the range which is not contained in any countable dimensional $G_{\delta}$ subset. We comment further on this phenomenon in $\S 5$.

Corollary 5.7 is another consequence of Theorem 5.1 which extends results in [K2]. Corollary 5.7 implies that a cell-like map $f: X \rightarrow Y$ from a metrizable space $X$ to a metrizable space $Y$ is a hereditary shape equivalence if $Y=\bigcup_{n=1}^{\infty} K_{n}$ where for each $n \geq 1, K_{n}$ is a closed subset of $Y$ and $f \mid f^{-1}\left(K_{n}\right): f^{-1}\left(K_{n}\right) \rightarrow K_{n}$ is a hereditary shape equivalence.

Another question which arises naturally from consideration of Theorem 1.1 and the Taylor example is the following. What sort of properties does the image of an ANR under a cell-like map have even if the map is not a fine homotopy equivalence? A variety of answers have been provided, such as Corollary 3.2 in [L2]. The answer provided by Theorem 5.9 contains and extends these results. It says that the image of a metrizable space under a $U V^{\infty}$ map is an ANE for the class of countable dimensional metrizable spaces. It also says that if the image space is itself countable dimensional, then it is an ANR.

If Theorem 5.9 is specialized to the identity map of a locally contractible space, it yields an interesting corollary. Corollary 5.10 says that every locally contractible metrizable space is an ANE for the class of countable dimensional metrizable spaces, and that every locally contractible countable dimensional metrizable space is an ANR. This corollary was actually discovered independently and earlier via different techniques by D. F. Addis and J. H. Gresham in [AG, G]. Their work extended a theorem of Haver [H3] to the effect that a locally contractible metrizable space is an ANR if it is the union of a countable number of finite dimensional compacta. Haver's work was no doubt motivated by two classical results in Borsuk. One result states that a locally contractible finite dimensional metrizable space is an ANR [Hu, Theorem V.7.1, p. 168]. The other is Borsuk's example of a locally contractible compact metrizable space which is not an ANR [Hu, pp. 169-172]. Clearly this space cannot be countable dimensional. (Borsuk's example is discussed further in $\S 4$ under the designation $\mathfrak{B}$ ).

We remind the reader that many of the results of $\S 5$ can be generalized by replacing the hypothesis of countable dimensionality by property $\mathrm{C}$. This is discussed in Appendix C.

2. Slice-trivial relations. The concept of slice-triviality is central to the developments in this paper. In this section we define slice-triviality and present three fundamental properties of slice-trivial relations. A slice-trivial relation can be arbitrarily closely approximated by maps (2.2). Furthermore, the maps approximating 
a slice-trivial relation are unique up to homotopy (2.3). Finally, a slice-trivial relation has an important "neigborhood extension property" (2.4).

Let $X$ and $Y$ be topological spaces, let $V \subset U \subset X \times Y$, and let $g: \operatorname{dom} V \rightarrow Y$ be a function such that $g \subset U$. A slice-contraction of $V$ onto $g$ in $U$ is a homotopy $\phi: V \times[0,1]^{\circ} \rightarrow U$ such that $\phi_{0}=1 \mid V$ and for each $x \in X, \phi(V \mid x \times[0,1]) \subset U \mid x$ and $\phi_{1}(V \mid x)=g \mid x$. If there is a slice-contraction of $V$ onto $g$ in $U$, we say that $V$ slice-contracts or is slice-contractible onto $g$ in $U$.

2.1. Proposition. Let $X$ and $Y$ be topological spaces. Suppose that a subset $V$ of $X \times Y$ slice-contracts onto a function $g: \operatorname{dom} V \rightarrow Y$ in $X \times Y$. The function $g$ is continuous under either of the following two hypotheses.

(1) $V: X \rightarrow Y$ is a continuous relation with compact point images.

(2) $V$ is an open subset of $X \times Y$.

ProOF. Let $\phi: V \times[0,1] \rightarrow X \times Y$ be a slice-contraction of $V$ onto $g$ in $X \times Y$. Then $g=\pi \circ \phi_{1} \circ(1 \mid X, V) \circ i$ where $i$ : $\operatorname{dom} V \rightarrow X$ denotes inclusion and $\pi: X \times Y \rightarrow$ $Y$ denotes projection. Under hypothesis (1), the relation $(1 \mid X, V): X \rightarrow X \times Y$ is continuous by A.6. The continuity of $g$ is then a consequence of A.4. Under hypothesis (2), the continuity of $g$ is established by arguing that if 0 is an open subset of $V$, then $(1 \mid X, V)^{-1}(0)$ is an open subset of $X$. Indeed, this is the case because the projection $\tau: X \times Y \rightarrow X$ is an open map and $(1 \mid X, V)^{-1}(0)=\tau(0)$.

Let $R: X \rightarrow Y$ be a relation from a topological space $X$ to a topological space $Y$. $R: X \rightarrow Y$ is slice-trivial in $X \times Y$ if $R$ is continuous with compact point images and if each neighborhood $U$ of $R$ in $X \times Y$ contains a neighborhood $V$ of $R$ in $X \times Y$ such that $V$ slice-contracts in $U$. In those contexts where no confusion can arise, we shorten " $R: X \rightarrow Y$ is slice-trivial in $X \times Y$ " to " $R: X \rightarrow Y$ is slice-trivial". Notice that the definition of slice-triviality does not require $\operatorname{dom} R=X$. However, it does stipulate that $V$ be a neighborhood of $R$ in $X \times Y$, not just in $(\operatorname{dom} R) \times Y$. Slice-triviality is a slice-respecting global $U V^{\infty}$ property for the subset $R$ of $X \times Y$.

2.2. Proposition (APPROXIMABILITY). Let $R: X \rightarrow Y$ be a slice-trivial relation from a topological space $X$ to a topological space $Y$. Then every neighborhood of $R$ in $X \times Y$ contains a map from $\operatorname{dom} R$ to $Y$.

Moreover, if $X$ is a normal space, then every neighborhood $U$ of $R$ in $X \times Y$ contains a neighborhood $V$ of $R$ in $X \times Y$ with the following property. If $A$ and $B$ are closed subsets of $\operatorname{dom} R$ with $A \subset$ int $_{\operatorname{dom} R} B$, and if $f: B \rightarrow Y$ is a map with $f \subset V$, then $f \mid A$ extends to a map $F: \operatorname{dom} R \rightarrow Y$ with $F \subset U$.

Proof. If $U$ is a neighborhood of $R$ in $X \times Y$, then $R$ slice-contracts onto a function $g: \operatorname{dom} R \rightarrow Y$ in $U$. Hence $g \subset U$ and, by $2.1, g$ is continuous.

Now assume $X$ is a normal space, and let $U$ be a neighborhood of $R$ in $X \times Y$. Then there is an open neighborhood $V$ of $R$ in $X \times Y$ and a slice-contraction $\phi: V \times[0,1] \rightarrow U$ of $V$ onto a map $g: \operatorname{dom} V \rightarrow Y$ in $U$. Suppose $A$ and $B$ are closed subsets of $\operatorname{dom} R, A \subset \operatorname{int}_{\operatorname{dom} R} B, f: B \rightarrow Y$ is a map and $f \subset V$. Let $\pi: X \times Y \rightarrow Y$ denote the projection, and define the map

$$
\psi:(B \times[0,1]) \cup(\operatorname{dom} R \times\{1\}) \rightarrow Y
$$

by the formulas

$$
\begin{aligned}
& \psi(x, t)=\pi \circ \phi((x, f(x)), t) \text { for }(x, t) \in B \times[0,1] \\
& \psi(x, 1)=g(x) \text { for } x \in \operatorname{dom} R .
\end{aligned}
$$


Then $\psi(x, 0)=f(x)$ for each $x \in B$. Urysohn's Lemma [D, pp. 146-147] provides a map $\lambda: \operatorname{dom} R \rightarrow[0,1]$ such that $\lambda(A)=0$ and $\lambda(\operatorname{dom} R-B)=1$. Define the map

$$
\Lambda: \operatorname{dom} R \rightarrow(B \times[0,1]) \cup(\operatorname{dom} R \times\{1\})
$$

by $\Lambda(x)=(x, \lambda(x))$ for $x \in \operatorname{dom} R$. Finally the desired map $F$ : $\operatorname{dom} R \rightarrow Y$ such that $F|A=f| A$ and $F \subset U$ is defined by $F=\psi \circ \Lambda$.

2.3. Proposition (Homotopy Uniqueness). Let $R: X \rightarrow Y$ be a slicetrivial relation from a topological space $X$ to a topological space $Y$. Then every neighborhood $U$ of $R$ in $X \times Y$ contains a neighborhood $V$ of $R$ in $X \times Y$ with the following property. If $f, g: \operatorname{dom} R \rightarrow Y$ are maps with $f \cup g \subset V$, then there is a homotopy $h: \operatorname{dom} R \times[0,1] \rightarrow Y$ such that $h_{0}=f, h_{1}=g$ and $h_{t} \subset U$ for each $t \in[0,1]$.

Moreover, if $X$ is a normal space, then every neighborhood $U$ of $R$ in $X \times Y$ contains a neighborhood $V$ of $R$ in $X \times Y$ with the following property. If $A$ and $B$ are closed subsets of $\operatorname{dom} R$ with $A \subset \operatorname{int}_{\operatorname{dom} R} B, f, g: \operatorname{dom} R \rightarrow Y$ are maps with $f \cup g \subset V$, and $h: B \times[0,1] \rightarrow Y$ is a homotopy with $h_{0}=f\left|B, h_{1}=g\right| B$ and $h_{t} \subset V$ for each $t \in[0,1]$, then there is a homotopy $H: \operatorname{dom} R \times[0,1] \rightarrow Y$ such that $H_{0}=f, H_{1}=g, H|A \times[0,1]=h| A \times[0,1]$ and $H_{t} \subset U$ for each $t \in[0,1]$.

PROOF. If $U$ is a neighborhood of $R$ in $X \times Y$, then there is a neighborhood $V$ of $R$ in $X \times Y$ and a slice-contraction $\phi: V \times[0,1] \rightarrow U$ of $V$ in $U$. If $f, g: \operatorname{dom} R \rightarrow Y$ are maps with $f \cup g \subset V$, then we define a homotopy $h$ : $\operatorname{dom} R \times[0,1] \rightarrow Y$ such that $h_{0}=f, h_{1}=g$ and $h_{t} \subset U$ for each $t \in[0,1]$ as follows:

$$
\begin{aligned}
& h(x, t)=\pi \circ \phi((x, f(x)), 2 t) \quad \text { for }(x, t) \in \operatorname{dom} R \times\left[0, \frac{1}{2}\right], \\
& h(x, t)=\pi \circ \pi((x, g(x)), 2-2 t) \quad \text { for }(x, t) \in \operatorname{dom} R \times\left[\frac{1}{2}, 1\right] .
\end{aligned}
$$

Here $\pi: X \times Y \rightarrow Y$ denotes projection.

Now assume $X$ is a normal space, and let $U$ be a neighborhood of $R$ in $X \times Y$. Then there is an open neighborhood $V$ of $R$ in $X \times Y$ and a slice-contraction $\phi: V \times[0,1] \rightarrow U$ of $V$ onto a map $k: \operatorname{dom} V \rightarrow Y$ in $U$. Suppose $A$ and $B$ are closed subsets of dom $R$ with $A \subset$ int $_{\text {dom } R} B, f, g$ : $\operatorname{dom} R \rightarrow Y$ are maps with $f \cup g \subset V$, and $h: B \times[0,1] \rightarrow Y$ is a homotopy with $h_{0}=f\left|B, h_{1}=g\right| B$ and $h_{t} \subset V$ for each $t \in[0,1]$. Let

$$
P=(B \times[0,1] \times[0,1]) \cup(\operatorname{dom} R \times((\{0,1\} \times[0,1]) \cup([0,1] \times\{1\}))) .
$$

We define the map $\psi: P \rightarrow Y$ by the formulas

$$
\begin{array}{ll}
\psi(x, s, t)=\pi \circ \phi((x, h(x, s)), t) & \text { for }(x, s, t) \in B \times[0,1] \times[0,1], \\
\psi(x, 0, t)=\pi \circ \phi((x, f(x)), t) & \text { for } x \in \operatorname{dom} R \text { and } t \in[0,1], \\
\psi(x, 1, t)=\pi \circ \phi((x, g(x)), t) & \text { for } x \in \operatorname{dom} R \text { and } t \in[0,1], \\
\psi(x, s, 1)=k(x) & \text { for } x \in \operatorname{dom} R \text { and } s \in[0,1] .
\end{array}
$$

Again $\pi: X \times Y \rightarrow Y$ denotes projection. Then $\psi(x, s, 0)=h(x, s)$ for $(x, s) \in B \times$ $[0,1], \psi(x, 0,0)=f(x)$ and $\psi(x, 1,0)=g(x)$ for $x \in \operatorname{dom} R$, and $(x, \psi(x, s, t)) \in U$ for each $(x, s, t) \in P$.

The desired homotopy $H: \operatorname{dom} R \times[0,1] \rightarrow Y$ is defined as $H=\psi \circ \Lambda$, where $\Lambda: \operatorname{dom} R \times[0,1] \rightarrow P$ is a map such that $\Lambda(x, s)=(x, s, 0)$ for each $(x, s) \in$ $(A \times[0,1]) \cup(\operatorname{dom} R \times\{0,1\})$ and $\Lambda$ preserves first coordinates. To form $\Lambda$, we require 
two auxiliary maps. The first map $\lambda: \operatorname{dom} R \rightarrow[0,1]$ is provided by Urysohn's Lemma $[\mathbf{D}$, pp. 146-147] so that $\lambda(A)=0$ and $\lambda(\operatorname{dom} R-B)=1$. The second map $\mu:[0,1] \times[0,1] \rightarrow[0,1] \times[0,1]$ has the following properties: $\mu(s, 0)=(s, 0)$ for $s \in[0,1], \mu(\{0\} \times[0,1])=(0,0), \mu(\{1\} \times[0,1])=(1,0)$ and $\mu([0,1] \times\{1\})=$ $(\{0,1\} \times[0,1]) \cup([0,1] \times\{1\})$. Now $\Lambda$ is defined by $\Lambda(x, s)=(x, \mu(s, \lambda(x)))$ for $(x, s) \in \operatorname{dom} R \times[0,1]$. We leave to the reader the verification that $\Lambda$ and $H=\psi \circ \Lambda$ have the desired properties.

2.4. Proposition (The Neighborhood Extension Property). Let $X$ and $Y$ be metrizable spaces. If $R: X \rightarrow Y$ is a slice-trivial relation, then there is a continuous relation $S: X \rightarrow Y$ such that $\operatorname{dom} R \subset \operatorname{int}(\operatorname{dom} S), S \mid \operatorname{dom} R=R$ and $S \mid(\operatorname{dom} S-\operatorname{dom} R)$ is a function.

PROOF. If $\tau$ is a metric on a space $Z, A \subset Z$ and $\varepsilon>0$, we use the notation " $N_{\tau}(A, \varepsilon)$ " to denote the open $\varepsilon$-neighborhood of $A$ in $Z$ with respect to $\tau$ :

$$
N_{\tau}(A, \varepsilon)=\{z \in Z: \tau(a, z)<\varepsilon \text { for some } a \in A\} .
$$

Let $\rho$ and $\sigma$ be metrics on $X$ and $Y$, respectively. Define the metric $\tau$ on $X \times Y$ by

$$
\tau\left((x, y),\left(x^{\prime}, y^{\prime}\right)\right)=\max \left\{\rho\left(x, x^{\prime}\right), \sigma\left(y, y^{\prime}\right)\right\}
$$

for $(x, y)$ and $\left(x^{\prime}, y^{\prime}\right) \in X \times Y$.

Let $U_{0}=X \times Y$. For each $i \geq 1$, there is an open neighborhood $U_{i}$ of $R$ in $X \times Y$ such that $U_{i} \subset N_{\tau}(R, 1 / i)$ and a slice-contraction $\phi_{i}: U_{i} \times[0,1] \rightarrow U_{i-1}$ of $U_{i}$ onto a map $g_{i}: \operatorname{dom} U_{i} \rightarrow Y$ in $U_{i-1}$.

Let $A_{0}=X$. For each $i \geq 1$, let $A_{i}$ be a closed subset of $X$ such that $\operatorname{dom} R \subset$ $\operatorname{int} A_{i} \subset A_{i} \subset\left(\operatorname{dom} U_{i}\right) \cap\left(\operatorname{int} A_{i-1}\right)$. Then $\operatorname{dom} R=\bigcap_{i=1}^{\infty} A_{i}$, because $A_{i} \subset$ $\operatorname{dom} U_{i} \subset N_{\rho}(\operatorname{dom} R, 1 / i)$ for each $i \geq 1$, and $\operatorname{dom} R=R^{-1}(Y)$ is a closed subset of $X$.

For each $i \geq 0$, let $B_{i}=A_{i}-\operatorname{int} A_{i+1}$. Then $A_{2}=(\operatorname{dom} R) \cup\left(\bigcup_{i=2}^{\infty} B_{i}\right)$. For each $i \geq 2$, Urysohn's Lemma $\left[\mathbf{D}\right.$, pp. 146-147] provides a map $\lambda_{i}: B_{i} \rightarrow[0,1]$ such that $\lambda_{i}\left(B_{i} \cap B_{i+1}\right)=0$ and $\lambda_{i}\left(B_{i-1} \cap B_{i}\right)=1$.

For each $i \geq 2$, define the map $h_{i}: B_{i} \rightarrow Y$ by the formula

$$
h_{i}(x)=\pi \circ \phi_{i-1}\left(\left(x, g_{i}(x)\right), \lambda_{i}(x)\right)
$$

for $x \in B_{i}$. Here $\pi: X \times Y \rightarrow Y$ denotes projection. It is easy to verify that $h_{i} \subset U_{i-2}, h_{i}=g_{i}$ on $B_{i} \cap B_{i+1}$ and $h_{i}=g_{i-1}$ on $B_{i-1} \cap B_{i}$ for each $i \geq 2$. Consequently $h_{i}=h_{i+1}$ on $B_{i} \cap B_{i+1}$ for each $i \geq 2$. As a result, we can define a map $h:\left(A_{2}-\operatorname{dom} R\right) \rightarrow Y$ by setting $h \mid B_{i}=h_{i}$ for each $i \geq 2$.

The desired relation $S: X \rightarrow Y$ is defined by $S=R \cup h$. Thus $\operatorname{dom} S=A_{2}$, and $S \mid A_{i} \subset U_{i-2}$ for each $i \geq 2$.

The properties required of $S$ in the conclusion of this proposition are all immediate except for the continuity of $S$ at points of $\operatorname{dom} R$. To prove this, let $x \in \operatorname{dom} R$ and let $M$ be a neigborhood of $R(x)$ in $Y$. Choose $\varepsilon>0$ so that $N_{\sigma}(R(x), 2 \varepsilon) \subset M$. Next choose $\delta>0$ so that $R\left(N_{\rho}(x, 2 \delta)\right) \subset N_{\sigma}(R(x), \varepsilon)$. Finally choose $i \geq 1$ so that $1 / i \leq \min \{\delta, \varepsilon\}$. Let $L=N_{\rho}(x, \delta) \cap\left(\operatorname{int} A_{i+2}\right)$. Recall that $U_{i} \subset N_{\tau}(R, 1 / i)$. We leave it to the reader to prove that these choices guarantee that $U_{i}(L) \subset M$. Since $S \mid L \subset U_{i}$, we conclude that $S(L) \subset M$. This establishes the continuity of $S$ at points of $\operatorname{dom} R$. 
3. $U V^{\infty}$ relations. A relation $R: X \rightarrow Y$ from a topological space $X$ to a topological space $Y$ is a $U V^{\infty}$ relation if $R$ is continuous and if each point image of $R$ is either empty or a compact $U V^{\infty}$ subset of $Y$.

This section begins with the observation that a slice-trivial relation is $U V^{\infty}$. The rest of the section is devoted to a proof of the Main Theorem which gives sufficient conditions for a $U V^{\infty}$ relation to be slice-trivial.

Not all $U V^{\infty}$ relations are slice-trivial. Indeed, if $\tau: T \rightarrow \mathbf{Q}$ is Taylor's cell-like map which is not a shape equivalence $[\mathbf{T}]$, and $i: T \rightarrow \mathbf{Q}$ is an embedding, then $i \circ f^{-1}: \mathbf{Q} \rightarrow \mathbf{Q}$ is a $U V^{\infty}$ relation which is not slice-trivial, according to Theorem 4.5 .

3.1. PROposition. Let $R: X \rightarrow Y$ be a relation from a Hausdorff space $X$ to a topological space $Y$. If $R$ is slice-trivial, then it is $U V^{\infty}$.

Proof. Let $x \in \operatorname{dom} R$ and let $L$ be a neighborhood of $R(x)$ in $Y$. Then $U=(X \times L) \cup((X-\{x\}) \times Y)$ is a neighborhood of $R$ in $X \times Y$. Hence there is a neighborhood $V$ of $R$ in $X \times Y$ and a slice-contraction $\phi: V \times[0,1] \rightarrow U$ of $V$ in $U . M=V(x)$ is a neighborhood of $R(x)$ in $Y$. Since $L=U(x)$, a contraction $\psi: M \times[0,1] \rightarrow L$ of $M$ in $L$ is defined by the formula

$$
\psi(y, t)=\pi \circ \phi((x, y), t) \quad \text { for }(y, t) \in M \times[0,1] .
$$

Here $\pi: X \times Y \rightarrow Y$ denotes projection.

3.2. MAIN THEOREM. Let $R: X \rightarrow Y$ be a $U V^{\infty}$ relation from a metrizable space $X$ to a topological space $Y$. If there is a sequence $\left\{K_{i}\right\}$ of closed subsets of $\operatorname{dom} R$ such that:

(1) $\operatorname{dom} R-\bigcup_{i=1}^{\infty} K_{i}$ is countable dimensional, and

(2) $R \mid K_{i}$ is slice-trivial in $X \times Y$ for each $i \geq 1$, then $R$ is slice-trivial.

The proof of the Main Theorem rests on the following five lemmas. The first three lemmas reformulate the hypotheses of the Main Theorem in more useful terms. The fourth lemma plays a central role in the proof of the Main Theorem; it tells how to produce a single global slice-contraction from a sequence of appropriately nested local slice-contractions. The fifth lemma is a device for extending slice-contractions which is crucial to the fourth lemma.

The first of the five lemmas establishes that a countable dimensional subset of a metrizable space has a certain covering property. (This covering property is intimately connected to property $\mathrm{C}$; this connection is elaborated in Appendix C.)

3.3. LEMMA. If $Z$ is a countable dimensional subset of a metrizable space $X$, then $Z$ has the following property. If for each $i \geq 1, \mathfrak{L}_{i}$ is a collection of open subsets of $X$ which covers $Z$, then there is a collection of open subsets of $X$ which covers $Z$ and which has form $\bigcup_{i=1}^{\infty} \mathfrak{M}_{i}$ where for each $i \geq 1$, the elements of $\mathfrak{M}_{i}$ are disjoint and $\mathfrak{M}_{i}$ refines $\mathfrak{L}_{i}$.

ProOF. $Z=\bigcup_{i=1}^{\infty} Z_{i}$ where each $Z_{i}$ is zero-dimensional. Fix $i \geq 1$. Given a collection $\mathfrak{L}_{i}$ of open subsets of $X$ which covers $Z_{i}$, there is a collection $\mathfrak{N}_{i}$ of (relatively) open subsets of $Z_{i}$ which covers $Z_{i}$ such that the elements of $\mathfrak{N}_{i}$ are 
disjoint and $\mathfrak{N}_{i}$ refines $\mathfrak{L}_{i}$. For each $N \in \mathfrak{N}_{i}$, choose $L(N) \in \mathfrak{L}_{i}$ so that $N \subset L(N)$, and define

$$
M(N)=L(N) \cap\{x \in X: \rho(x, N)<\rho(x, Z-N)\} .
$$

(Here $\rho$ is a metric on $X ; \rho(x, A)=\inf \{\rho(x, a): a \in A\}$ for $x \in X$ and $A \subset X$.) Finally, let $\mathfrak{M}_{i}=\left\{M(N): N \in \mathfrak{N}_{i}\right\}$; then $\mathfrak{M}_{i}$ is a collection of open subsets of $X$ which covers $Z_{i}$ such that the elements of $\mathfrak{M}_{i}$ are disjoint and $\mathfrak{M}_{i}$ refines $\mathfrak{L}_{i}$.

The second of the five lemmas uses the $U V^{\infty}$ hypothesis to cover the given relation with local slice-contractions in a uniform way.

3.4. LEMMA. Let $R: X \rightarrow Y$ be a $U V^{\infty}$ relation from a metrizable space $X$ to a topological space $Y$. Then for every open neighborhood $U$ of $R$ in $X \times Y$, there is an open neighborhood $V$ of $R$ in $X \times Y$ and a collection $\mathfrak{L}$ of open subsets of $X$ which covers dom $R$ such that $V \subset U$ and $V \mid L$ slice-contracts in $U$ for each $L \in \mathfrak{L}$.

Proof. Let $x \in \operatorname{dom} R$. Since $R \mid x$ is a compact subset of $U$, there are open neighborhoods $J(x)$ of $x$ in $X$ and $O(x)$ of $R(x)$ in $Y$ such that $J(x) \times O(x) \subset$ $U$. Since $R(x)$ is a $U V^{\infty}$ subset of $Y$, there is an open neighborhood $N(x)$ of $R(x)$ in $Y$ and a contraction $\phi_{x}: N(x) \times[0,1] \rightarrow O(x)$ of $N(x)$ in $O(x)$. Now a slice-contraction $\psi_{x}$ of $J(x) \times N(x)$ in $J(x) \times O(x)$ is defined by the formula $\psi_{x}((w, y), t)=\left(w, \phi_{x}(y, t)\right)$ for $(w, y) \in J(x) \times N(x)$ and $t \in[0,1]$.

For each $x \in X-\operatorname{dom} R$, let $J(x)=X-\operatorname{dom} R$ and let $N(x)=\emptyset$. We now invoke Enlargement Lemma A.8 to obtain an open cover $\{L(x): x \in X\}$ of $X$ and an open neighborhood $V$ of $R$ in $X \times Y$ such that $L(x) \subset J(x)$ and $V(L(x)) \subset N(x)$ for each $x \in X$. Then $V \mid L(x) \subset J(x) \times N(x)$ for each $x \in X$. So $V \subset U$. Let $\mathfrak{L}=\{L(x): x \in \operatorname{dom} R\}$. Then $\mathfrak{L}$ covers $\operatorname{dom} R$. Furthermore, if $L(x) \in \mathfrak{L}$, then $\psi_{x} \mid(V \mid L(x)) \times[0,1]$ is a slice-contraction of $V \mid L(x)$ in $U$.

3.5. LEMMA. Let $R: X \rightarrow Y$ be a continuous relation with compact point images from a metrizable space $X$ to a topological space $Y$. If $K$ is a closed subset of $X$ such that $R \mid K$ is slice-trivial in $X \times Y$, then for every open neighborhood $U$ of $R$ in $X \times Y$, there is an open neighborhood $V$ of $R$ in $X \times Y$ and a neighborhood $M$ of. $K$ in $X$ such that $V \subset U$ and $V \mid M$ slice-contracts in $U$.

ProOF. Let $W$ be an open neighborhood of $R \mid K$ in $X \times Y$ which slice-contracts in $U$. According to A.6, $(1 \mid X, R): X \rightarrow X \times Y$ is continuous. It follows that there is an open neighborhood $M$ of $K$ in $X$ such that $R \mid \operatorname{cl} M=(1 \mid X, R)(\operatorname{cl} M) \subset W$. Let $V=W \cup(U \mid(X-\operatorname{cl} M))$. Then $V$ is an open neighborhood of $R$ in $X \times Y$, $V \subset U$ and $V \mid M \subset W$.

As we mentioned earlier, the fourth lemma is the principal ingredient in the proof of the Main Theorem.

3.6. LEMMA. Let $R: X \rightarrow Y$ be a relation from a metrizable space $X$ to a topological space $Y$, and let $U_{0}$ be a neighborhood of $R$ in $X \times Y$. The following conditions guarantee that there is a neighborhood $V$ of $R$ in $X \times Y$ which slicecontracts in $U_{0}$. There is a sequence $\left\{U_{i}\right\}$ of open neighborhoods of $R$ in $X \times Y$ and there is a sequence $\left\{M_{i}\right\}$ of open subsets of $X$ such that $\left\{M_{i}\right\}$ covers $\operatorname{dom} R$, and $U_{i} \subset U_{i-1}$ and $U_{i} \mid M_{i}$ slice-contracts in $U_{i-1}$ for each $i \geq 1$.

The proof of this lemma requires the following device. 
3.7. Extension Lemma. Let $A, B$, and $C$ be closed subsets of a metrizable space $X$ such that $C \subset \operatorname{int} A$. Let $Y$ be a space and let $U \subset X \times Y, V \subset A \times Y$ and $W \subset B \times Y$. Suppose $\phi$ is a slice-contraction of $V$ in $U$ and $\psi$ is a slice-contraction of $W$ in $U$ such that $\psi((W \mid A \cap B) \times[0,1]) \subset V$. Then there is a slice-contraction $\chi$ of $(V \mid C) \cup W$ in $U$ such that $\chi=\phi$ on $(V \mid C) \times[0,1]$.

Proof. Let $P$ and $Q$ be closed subsets of $X$ such that

$$
C \subset \operatorname{int} P \subset P \subset \operatorname{int} Q \subset Q \subset \operatorname{int} A .
$$

Let $\lambda: X \rightarrow[0,1]$ and $\mu: X \rightarrow[0,1]$ be maps satisfying the conditions: $\lambda(P)=$ $0, \mu(X-Q)=0$ and $\lambda^{-1}(1) \cup \mu^{-1}(1)=X$. (For instance, let $\nu: X \rightarrow[0,2]$ be a map provided by Urysohn's Lemma [D, pp. 146-147] satisfying $\nu(P)=0$ and $\nu(X-Q)=2$; and define $\lambda(x)=\min \{\nu(x), 1\}$ and $\mu(x)=\min \{2-\nu(x), 1\}$ for each $x \in X$.)

Let $T=(V \mid C) \cup W$. Let $L=\operatorname{int} P \cap(C \cup B), M=(\operatorname{int} A-C) \cap B$ and $N=B-Q$. Then $\{L, M, N\}$ is an open cover of $C \cup B$. Define $\chi: T \times[0,1] \rightarrow U$ by the formulas

$$
\chi_{t}(x, y)= \begin{cases}\phi_{t}(x, y) & \text { for }(x, y) \in T \mid L \\ \phi_{\mu(x) t} \circ \psi_{\lambda(x) t}(x, y) & \text { for }(x, y) \in T \mid M \\ \psi_{t}(x, y) & \text { for }(x, y) \in T \mid N\end{cases}
$$

for each $t \in[0,1]$. We leave to the reader the straightforward verification that $\chi$ is well defined and has the desired properties.

PROOF OF LEMMA 3.6. We begin by "shrinking" the open cover $\left\{M_{i}\right\} \cup$ $\{X-\operatorname{dom} R\}$ of $X([\mathbf{D}]$, Theorem VII.6.1, p. 152 and Theorem VIII.1.4, p. 162) to obtain a sequence $\left\{H_{i}\right\}$ of closed subsets of $X$ such that int $\left.H_{i}\right\}$ covers dom $R$ and $H_{i} \subset M_{i}$ for each $i \geq 1$. Next for each $i \geq 1$ we choose a sequence $\left\{K_{i, j}: j \geq 1\right\}$ of closed subsets of $X$ such that

$$
H_{i} \subset \operatorname{int} K_{i, j} \subset K_{i, j} \subset M_{i} \quad \text { and } \quad K_{i, j+1} \subset \operatorname{int} K_{i, j}
$$

for each $j \geq 1$.

We define two sequences $\left\{V_{j}\right\}$ and $\left\{T_{j}\right\}$ of subsets of $X \times Y$ by the formulas

$$
V_{j}=\bigcup_{i=1}^{j} U_{i} \mid K_{i, j} \quad \text { and } T_{j}=\bigcup_{i=1}^{j} U_{i} \mid K_{i, j+1}
$$

for $j \geq 1$. Then $T_{j} \subset V_{j} \cap V_{j+1}$ for each $j \geq 1$.

For each $j \geq 1$, let $\psi_{j}$ be a slice-contraction of $U_{j} \mid M_{j}$ in $U_{j-1}$. By repeated application of Extension Lemma 3.7, we shall construct for each $j \geq 1$ a slicecontraction $\phi_{j}$ of $V_{j}$ in $U_{0}$ such that $\phi_{j}=\phi_{j+1}$ on $T_{j} \times[0,1]$.

We begin by setting $\phi_{1}=\psi_{1} \mid V_{1} \times[0,1]$. This is possible because $V_{1} \subset U_{1} \mid M_{1}$.

Let $j \geq 1$ and assume we have a slice-contraction $\phi_{j}$ of $V_{j}$ in $U_{0}$. We apply Extension Lemma 3.7 with the following substitutions: substitute $\bigcup_{i=1}^{j} K_{i, j}$ for $A, K_{j+1, j+1}$ for $B, \bigcup_{i=1}^{j} K_{i, j+1}$ for $C, U_{0}$ for $U, V_{j}$ for $V, U_{j+1} \mid K_{j+1, j+1}$ for $W, \phi_{j}$ for $\phi$, and $\psi_{j+1} \mid\left(U_{j+1} \mid K_{j+1, j+1}\right) \times[0,1]$ for $\psi$. Since

$$
\psi_{j+1} \mid\left(U_{j+1} \mid\left(\bigcup_{i=1}^{j} K_{i, j}\right) \cap K_{j+1, j+1}\right) \times[0,1]
$$


is a slice-contraction in $U_{j} \mid\left(\bigcup_{i=1}^{j} K_{i, j}\right) \cap K_{j+1, j+1}$, and the latter set is contained in $V_{j}$, then the hypotheses of the Extension Lemma hold. Hence, there is a slice-contraction $\chi$ of $\left(V_{j} \mid \bigcup_{i=1}^{j} K_{i, j+1}\right) \cup\left(U_{j+1, j+1}\right)$ in $U_{0}$ such that $\chi=\phi_{j}$ on $\left(V_{j} \mid \bigcup_{i=1}^{j} K_{i, j+1}\right) \times[0,1]$. Since

$$
V_{j+1} \subset\left(V_{j} \mid \bigcup_{i=1}^{j} K_{i, j+1}\right) \cup\left(U_{j+1} \mid K_{j+1, j+1}\right)
$$

and $T_{j} \subset V_{j} \mid \bigcup_{i=1}^{j} K_{i, j+1}$, then the desired slice-contraction $\phi_{j+1}$ of $V_{j+1}$ in $U_{0}$ satisfying $\phi_{j+1}=\phi_{j}$ on $T_{j} \times[0,1]$ is obtained by setting $\phi_{j+1}=\chi \mid V_{j+1} \times[0,1]$.

We define an open neighborhood $V$ of $R$ in $X \times Y$ by $V=\bigcup_{i=1}^{\infty}\left(U_{i} \mid\right.$ int $\left.H_{i}\right)$. A slice-contraction $\phi$ of $V$ in $U_{0}$ is defined by specifying that $\phi=\phi_{j}$ on

$$
\bigcup_{i=1}^{j}\left(U_{i} \mid \operatorname{int} H_{i}\right) \times[0,1] \text { for each } j \geq 1 \text {. }
$$

$\phi$ is well defined because $\bigcup_{i=1}^{j}\left(U_{i} \mid\right.$ int $\left.H_{i}\right) \subset T_{j}$ and $\phi_{j}=\phi_{j+1}$ on $T_{j} \times[0,1]$ for each $j \geq 1$. $\phi$ is continuous because $\bigcup_{i=1}^{j}\left(U_{i} \mid \operatorname{int} H_{i}\right)$ is an open subset of $V$ for each $j \geq 1$.

PROOF OF MAIN ThEOREM 3.2. Let $U_{0}$ be an open neighborhood of $R$ in $X \times Y$. Our plan is to achieve the conditions in the hypothesis of Lemma 3.6.

We alternatively invoke Lemmas 3.4 and 3.5 to obtain

(1) a sequence $\left\{U_{i}\right\}$ of open neighborhoods of $R$ in $X \times Y$,

(2) a sequence $\left\{\mathfrak{L}_{2 i-1}\right\}$ of collections of open subsets of $X$ each of which covers $\operatorname{dom} R$, and

(3) a sequence $\left\{M_{2 i}\right\}$ of open subsets of $X$

such that for each $i \geq 1, U_{i} \subset U_{i-1}, U_{2 i-1} \mid L$ slice-contracts in $U_{2 i-2}$ for each $L \in \mathfrak{L}_{2 i-1}, K_{i} \subset M_{2 i}$, and $U_{2 i} \mid M_{2 i}$ slice-contracts in $U_{2 i-1}$.

Let $Z=X-\bigcup_{i=1}^{\infty} K_{i}$. Since $Z$ is countable dimensional, Lemma 3.3 provides a collection of open subsets of $X$ which covers $Z$ and which has form $\bigcup_{i=1}^{\infty} \mathfrak{M}_{2 i-1}$ where for each $i \geq 1$, the elements of $\mathfrak{M}_{2 i-1}$ are disjoint and $\mathfrak{M}_{2 i-1}$ refines $\mathfrak{L}_{2 i-1}$. For each $i \geq 1$, let $M_{2 i-1}=\bigcup \mathfrak{M}_{2 i-1}$; it follows that $U_{2 i-1} \mid M_{2 i-1}$ slice-contracts in $U_{2 i-2}$.

Now $\left\{M_{i}\right\}$ is a collection of open subsets of $X$ which covers dom $R$, and $U_{i} \mid M_{i}$ slice-contracts in $U_{i-1}$ for each $i \geq 1$. To complete the proof, we appeal to Lemma 3.6.

4. Slice-triviality, fine homotopy equivalence and hereditary shape equivalence. The study of cell-like maps has given birth to the notions of fine homotopy equivalence and hereditary shape equivalence. After recalling their definitions, we shall develop connections between these concepts and slice-triviality. Our ultimate goal here is to make the application of Main Theorem 3.2 to the study of cell-like maps as easy as possible. The principal result of this section is Theorem 4.5. The four results which precede Theorem 4.5 have some independent interest beyond the role which they play in the proof of 4.5. Following the proof of Theorem 4.5 , we raise a question about spaces whose identity map is slice-trivial.

Let $X$ and $Y$ be topological spaces and let $f, g: X \rightarrow Y$ be maps. If $f$ is homotopic to $g$, we write $f \sim g$; and if $h: X \times[0,1] \rightarrow Y$ is a homotopy from $f$ to $g$ ( $h_{0}=f$ 
and $h_{1}=g$ ), then we write $h: f \sim g$. Let $\mathfrak{L}$ be a collection of subsets of $Y$. An $\mathfrak{L}$-homotopy from $f$ to $g$ is a homotopy $h: X \times[0,1] \rightarrow Y$ from $f$ to $g$ such that $\{h(\{x\} \times[0,1]): x \in X\}$ refines $\mathfrak{L}$. If there is an $\mathfrak{L}$-homotopy from $f$ to $g$, we say that $f$ is $\mathfrak{L}$-homotopic to $g$ and we write $f \sim g[\mathfrak{L}]$. If $h$ is an $\mathfrak{L}$-homotopy from $f$ to $g$, we write $h: f \sim g[\mathfrak{L}]$.

If $f: X \rightarrow Y$ is a function and $\mathfrak{L}$ is a collection of subsets of $Y$, we let $f^{-1} \mathfrak{L}=$ $\left\{f^{-1}(L): L \in \mathfrak{L}\right\}$.

A map $f: X \rightarrow Y$ from a topological space $X$ to a topological space $Y$ is a fine homotopy equivalence if for every open cover $\mathfrak{L}$ of $Y$, there is a map $g: Y \rightarrow X$ such that $f \circ g \sim 1 \mid Y[\mathfrak{L}]$ and $g \circ f \sim 1 \mid X\left[f^{-1} \mathfrak{L}\right]$.

We now recall Kozlowski's definitions of shape equivalence and hereditary shape equivalence [K2]. Let $X$ and $Y$ be topological spaces. If $f: X \rightarrow Y$ is a map, let $[f]$ denote the equivalence class of maps from $X$ to $Y$ which are homotopic to $f$. Let $[X, Y]$ denote the collection of all such equivalence classes of maps from $X$ to $Y$. If $f: X \rightarrow Y$ is a map and $Z$ is a topological space, then $f$ induces a function $f^{\#}:[Y, Z] \rightarrow[X, Z]$ which is defined by $f^{\#}([g])=[g \circ f]$ for each $[g] \in$ $[Y, Z]$. A map $f: X \rightarrow Y$ is a shape equivalence if for every ANR $Z$, the function $f^{\#}:[Y, Z] \rightarrow[X, Z]$ is a bijection. A map $f: X \rightarrow Y$ is a hereditary shape equivalence if $f \mid f^{-1} B: f^{-1} B \rightarrow B$ is a shape equivalence for every closed subset $B$ of $Y$.

The following definitions are of use in the next few results.

If $\mathfrak{L}$ is a collection of subsets of $Y$ and if $f, g: X \rightarrow Y$ are functions such that $\{\{f(x), g(x)\}: x \in X\}$ refines $\mathfrak{L}$, then we say that $f$ is within $\mathfrak{L}$ of $g$.

A map $f: X \rightarrow Y$ from a topological space $X$ to a topological space $Y$ is a fine homotopy injection if for every open cover $\mathfrak{L}$ of $Y$, there is a map $g: Y \rightarrow X$ such that $g \circ f \sim 1 \mid X\left[f^{-1} \mathfrak{L}\right]$.

4.1. THEOREM. Let $f: X \rightarrow Y$ be a proper onto map from a metrizable space $X$ to a metrizable space $Y$. If $f^{-1}: Y \rightarrow X$ is slice-trivial, then $f$ is a fine homotopy equivalence.

The proof has three steps.

Step 1. $f$ is a fine homotopy injection.

PROOF. Let $\mathfrak{L}$ be an open cover of $Y$. Corollary A.9 to the Enlargement Lemma provides a neighborhood $U$ of $f^{-1}$ in $Y \times X$ such that $\{U(y): y \in Y\}$ refines $f^{-1} \mathfrak{L}$. Since $f^{-1}$ is slice-trivial, there is a slice-contraction $\phi: f^{-1} \times[0,1] \rightarrow U$ of $f^{-1}$ onto a map $g: Y \rightarrow X$ in $U$. Let $\pi: Y \times X \rightarrow X$ denote projection, and define an $f^{-1} \mathfrak{L}$-homotopy $h: X \times[0,1] \rightarrow X$ from $1 \mid X$ to $g \circ f$ by the formula $h(x, t)=\pi \circ \phi((f(x), x), t)$ for $(x, t) \in X \times[0,1]$.

Step 2. For every open cover $\mathfrak{L}$ of $Y$, there is an open cover $\mathfrak{M}$ of $Y$ with the following property. If $g, h: Y \rightarrow X$ are maps such that both $g \circ f$ and $h \circ f$ are within $f^{-1} \mathfrak{M}$ of $1 \mid X$, then $g \sim h\left[f^{-1} \mathfrak{L}\right]$.

PROOF. Let $\mathfrak{L}$ be an open cover of $Y$. Corollary A.9 provides a neighborhood $U$ of $f^{-1}$ in $Y \times X$ such that $\{U(y): y \in Y\}$ refines $f^{-1} \mathfrak{L}$. According to Proposition 2.3, $U$ contains a neighborhood $V$ of $f^{-1}$ in $Y \times X$ with the following property. If $g, h: Y \rightarrow X$ are maps with $g \cup h \subset V$, then there is a homotopy $k: Y \times[0,1] \rightarrow X$ such that $k_{0}=g, k_{1}=h$ and $k_{t} \subset U$ for each $t \in[0,1]$. It follows that $k$ is an $f^{-1} \mathfrak{L}$-homotopy from $g$ to $h$.

Since $f^{-1}: Y \rightarrow X$ is a continuous relation with compact point images, there is an open cover $\mathfrak{M}$ of $Y$ such that $M \times f^{-1} M \subset V$ for each $M \in \mathfrak{M}$. Now if 
$g, h: Y \rightarrow X$ are maps such that $g \circ f$ and $h \circ f$ are within $f^{-1} \mathfrak{M}$ of $1 \mid X$, then

$$
g \cup h \subset \bigcup\left\{M \times f^{-1} M: M \in \mathfrak{M}\right\} \subset V ;
$$

and consequently $g \sim h\left[f^{-1} \mathfrak{L}\right]$.

Step 3. $f$ is a fine homotopy equivalence.

PROOF. Let $\mathfrak{L}_{0}$ to be an open cover of $Y$. We repeatedly use Steps 1 and 2 (and A.10) to obtain a sequence $\left\{\mathfrak{L}_{i}\right\}$ of open covers of $Y$ and a sequence $\left\{g_{i}\right\}$ of maps from $Y$ to $X$ with the following four properties.

(1) $\mathfrak{L}_{i}$ star-refines $\mathfrak{L}_{i-1}$ for each $i \geq 1$.

(2) $\operatorname{diam} L<1 / i$ for each $L \in \mathfrak{L}_{i}$, for each $i \geq 1$.

(3) If $g, h: Y \rightarrow X$ are maps such that both $g \circ f$ and $h \circ f$ are within $f^{-1} \mathfrak{L}_{i}$ of $1 \mid X$, then $g \sim h\left[f^{-1} \mathfrak{L}_{i-1}\right]$, for each $i \geq 1$.

(4) $g_{i} \circ f \sim 1 \mid X\left[f^{-1} \mathfrak{L}_{i}\right]$ for each $i \geq 1$.

Let $i \geq 1$. (1) and (4) imply that both $g_{i} \circ f$ and $g_{i+1} \circ f$ are within $f^{-1} \mathfrak{L}_{i}$ of $1 \mid X$. Hence (3) provides an $f^{-1} \mathfrak{L}_{i-1}$-homotopy from $g_{i}$ to $g_{i+1}$. Composing this homotopy with $f$ yields an $\mathfrak{L}_{i-1}$-homotopy from $f \circ g_{i}$ to $f \circ g_{i+1}$. Since $g_{i} \circ f$ is within $f^{-1} \mathfrak{L}_{i}$ of $1 \mid X$ and $f$ is onto, it also follows that $f \circ g_{i}$ is within $\mathfrak{L}_{i}$ of $1 \mid Y$.

(2) implies that the sequence $\left\{f \circ g_{i}\right\}$ converges uniformly to $1 \mid Y$. Hence the sequence of homotopies $f \circ g_{i} \sim f \circ g_{i+1}\left[\mathfrak{L}_{i-1}\right](i \geq 2)$ can be strung together to give a homotopy from $f \circ g_{2}$ to $1 \mid Y$; moreover, (1) implies this homotopy is an $\mathfrak{L}_{0}$-homotopy. (Indeed, condition (1) implies that whenever $2 \leq m<n$, the homotopies $f \circ g_{i} \sim f \circ g_{i+1}\left[\mathfrak{L}_{i-1}\right]$ for $m \leq i<n$ string together to yield an $\mathfrak{L}_{m-2^{-}}$ homotopy from $f \circ g_{m}$ to $f \circ g_{n}$.) Hence, $f \circ g_{2} \sim 1 \mid Y\left[\mathfrak{L}_{0}\right]$. On the other hand, (1) and (4) imply that $g_{2} \circ f \sim 1 \mid X\left[\mathfrak{L}_{0}\right]$.

4.2. Proposition. Let $f: X \rightarrow Y$ be a proper onto map from an $A N R X$ to a metrizable space $Y$. If $f$ is a fine homotopy injection, then $f^{-1}: Y \rightarrow X$ is slice-trivial.

PROOF. We shall argue that $f^{-1}$ is slice-contractible in each of its neighborhoods in $Y \times X$. Then the slice-triviality of $f^{-1}$ will follow from Lemma 4.3.

Let $U$ be a neighborhood of $f^{-1}$ in $Y \times X$. Since $f^{-1}: Y \rightarrow X$ is a continuous relation with compact point images, there is an open cover $\mathfrak{L}$ of $Y$ such that $L \times$ $f^{-1} L \subset U$ for each $L \in \mathfrak{L}$. Since $f$ is a fine homotopy injection, there is a map $g: Y \rightarrow X$ and an $f^{-1} \mathfrak{L}$-homotopy $h: X \times[0,1] \rightarrow X$ from $1 \mid X$ to $g \circ f$. A slice-contraction $\phi: f^{-1} \times[0,1] \rightarrow Y \times X$ of $f^{-1}$ onto $g$ in $X \times Y$ is defined by the formula

$$
\phi((f(x), x), t)=(f(x), h(x, t)) \quad \text { for }(x, t) \in X \times[0,1] .
$$

It follows that for each $x \in X$, there is an $L \in \mathfrak{L}$ such that $\phi(\{(f(x), x)\} \times[0,1]) \subset$ $L \times f^{-1} L$. Therefore, $\phi$ is a slice-contraction of $f^{-1}$ onto $g$ in $U$.

4.3. LEMMA. Let $R: X \rightarrow Y$ be a continuous relation with compact point images from a metrizable space $X$ to an ANR $Y$. If $R$ is slice-contractible in each of its neighborhhoods in $X \times Y$, then $R$ is slice-trivial in $X \times Y$.

Proof. Let $U$ be a neighborhood of $R$ in $X \times Y$. Then there is a slicecontraction $\phi: R \times[0,1] \rightarrow U$ of $R$ onto a map $g: \operatorname{dom} R \rightarrow Y$ in $U$. Our goal is to extend $\phi$ to a slice-contraction of a neighborhood of $R$ in $U$. 
Since $Y$ is an ANR, $G$ extends to a map $g: L \rightarrow Y$ where $L$ is a neighborhood of dom $R$ in $X$. Let $\pi: X \times Y \rightarrow Y$ denote projection, and define the map $\varsigma:(R \times$ $[0,1]) \cup((L \times Y) \times\{0,1\}) \rightarrow Y$ by the formulas

$$
\begin{array}{ll}
\varsigma((x, y), t)=\pi \circ \phi((x, y), t) & \text { for }((x, y), t) \in R \times[0,1], \\
\varsigma((x, y), 0)=y & \text { for }(x, y) \in L \times Y, \\
\varsigma((x, y), 1)=G(x) & \text { for }(x, y) \in L \times Y .
\end{array}
$$

Since the domain of $\zeta$ is a closed subset of $(L \times Y) \times[0,1]$ (using A.7), and since $Y$ is an ANR, then $\zeta$ extends to a map $\zeta^{+}:(W \times[0,1]) \cup((L \times Y) \times\{0,1\}) \rightarrow Y$ where $W$ is a neighborhood of $R$ in $L \times Y$.

Finally, a slice-contraction $\psi: W \times[0,1] \rightarrow X \times Y$ of $W$ onto $G \mid \operatorname{dom} W$ in $X \times Y$ is defined by $\psi((x, y), t)=\left(x, \varsigma^{+}((x, y), t)\right)$ for $((x, y), t) \in W \times[0,1]$. Also $\psi \mid R \times[0,1]=\phi$. So $\psi(R \times[0,1]) \subset U$. Hence $W$ contains a neigborhood $V$ of $R$ in $X \times Y$ such that $\psi(V \times[0,1]) \subset U$. We conclude that $\psi \mid V \times[0,1]$ is a slice-contraction of $V$ onto $G \mid \operatorname{dom} V$ in $U$.

4.4. Corollary. If $f: X \rightarrow Y$ is a proper onto map from an ANR $X$ to a metrizable space $Y$, then the following three statements are equivalent.

(1) $f$ is a fine homotopy injection.

(2) $f$ is a fine homotopy equivalence.

(3) $f^{-1}: Y \rightarrow X$ is slice-trivial.

The following terminology is used in Theorem 4.5.

Let $f: X \rightarrow Y$ be a proper onto map from a metrizable space $X$ to a metrizable space $Y$. A trivial extension of $f$ is a proper onto map $f_{+}: X_{+} \rightarrow Y_{+}$from a metrizable space $X_{+}$to a metrizable space $Y_{+}$to which are associated closed embeddings $i: X \rightarrow X_{+}$and $j: Y \rightarrow Y_{+}$such that $f_{+} \circ i=j \circ f$ and $f_{+}$maps $X_{+}-i(X)$ homeomorphically onto $Y_{+}-j(Y)$. An ANR trivial extension of $f$ is a trivial extension $f_{+}: X_{+} \rightarrow Y_{+}$of $f$ in which $X_{+}$is an ANR. Given a closed embedding $i: X \rightarrow X_{+}$, there is an obvious trivial extension $f_{+}: X_{+} \rightarrow Y_{+}$of $f$ called the trivial extension of $f$ by $i$, which is obtained as follows. Let $Y_{+}=X_{+} \cup_{f \circ i^{-1}} Y$, the space obtained by attaching $X_{+}$to $Y$ via the map $f \circ i^{-1}: i(X) \rightarrow Y$. Let $j: Y \rightarrow Y_{+}$be the natural "inclusion", and let $f_{+}: X_{+} \rightarrow Y_{+}$be the natural map such that $f_{+} \circ i=j \circ f$ and $f_{+}$is the "identity" on $X_{+}-i(X)$.

Let $R: X \rightarrow Y$ be a relation from a set $X$ to a topological space $Y$. An open cover of $R$ is a collection of open subsets of $Y$ which is refined by $\{R(x): x \in X\}$.

4.5. THEOREM. If $f: X \rightarrow Y$ is a proper onto map from a metrizable space $X$ to a metrizable space $Y$, then the following nine conditions are equivalent.

(1a) There is a closed embedding $i: X \rightarrow X_{+}$into a metrizable space $X_{+}$such that for every open cover $\mathfrak{M}$ of $i \circ f^{-1}: Y \rightarrow X_{+}$, there is a map $g: Y \rightarrow X_{+}$such that $g \circ f \sim i[\mathfrak{M}]$.

(1b) For every map $\phi: X \rightarrow Z$ into an $A N R Z$, for every open cover $\mathfrak{M}$ of $\phi \circ f^{-1}: Y \rightarrow Z$, there is a map $g: Y \rightarrow Z$ such that $g \circ f \sim \phi[\mathfrak{M}]$.

(2a) There is a closed embedding $i: X \rightarrow X_{+}$into a metrizable space $X_{+}$such that $i \circ f^{-1}: Y \rightarrow X_{+}$is slice-trivial.

(2b) For every closed embedding $i: X \rightarrow X_{+}$into an $A N R X_{+}, i \circ f^{-1}: Y \rightarrow X_{+}$ is slice-trivial.

(3a) The inverse of some trivial extension of $f$ is slice-trivial. 
(3b) The inverse of every ANR trivial extension of $f$ is slice-trivial.

(4a) Some trivial extension of $f$ is a fine homotopy equivalence.

(4b) Every ANR trivial extension of $f$ is a fine homotopy equivalence.

(5) $f$ is a hereditary shape equivalence.

The following diagrams indicate the logical order of the proof.

$$
\begin{aligned}
& (1 \mathrm{a}) \rightarrow(1 \mathrm{~b}) \rightarrow(3 \mathrm{~b}) \rightarrow(2 \mathrm{~b}) \\
& (3 \mathrm{~b}) \rightarrow(4 \mathrm{~b}) \rightarrow(3 \mathrm{a}) \rightarrow(2 \mathrm{a}) \rightarrow \\
& (4 \mathrm{a}) \rightarrow(5) \rightarrow(1 \mathrm{~b})
\end{aligned}
$$

We remark that the proof of Theorem 4.5 is essentially self-contained with the exception of the proof that (5) implies (1b). Here we rely on some deep work of Kozlowski.

Proof that (1a) implies (1b). Assume (1a). Let $\phi: X \rightarrow Z$ be a map into an ANR $Z$ and let $\mathfrak{M}$ be an open cover of $\phi \circ f^{-1}: Y \rightarrow Z$. Since $Z$ is an ANR, there is an open neighborhood 0 of $i(X)$ in $X_{+}$and a map $\psi: 0 \rightarrow Z$ such that $\psi \circ i=\phi$. Since $\psi^{-1} \mathfrak{M}$ is an open cover of $i \circ f^{-1}: Y \rightarrow X_{+}$, then (1a) provides a map $g: Y \rightarrow X_{+}$and a $\psi^{-1} \mathfrak{M}$-homotopy $h: X \times[0,1] \rightarrow X_{+}$from $i$ to $g \circ f$. It follows that $h(X \times[0,1]) \cup g(Y) \subset 0$. Hence we have a map $\psi \circ g: Y \rightarrow Z$ and an $\mathfrak{M}$-homotopy $\psi \circ h: X \times[0,1] \rightarrow Z$ from $\phi$ to $(\psi \circ g) \circ f$.

Proof that (1b) implies (3b). Assume (1b). Let $f_{+}: X_{+} \rightarrow Y_{+}$be an ANR trivial extension of $f$ with associated closed embeddings $i: X \rightarrow X_{+}$and $j: Y \rightarrow Y_{+}$. We shall argue that $f_{+}$is a fine homotopy injection. Then the slice-triviality of $f_{+}^{-1}: Y_{+} \rightarrow X_{+}$will follow from Proposition 4.2.

Let $\mathfrak{L}$ be an open cover of $Y_{+}$. (1b) provides a map $g: Y \rightarrow X_{+}$and an $f_{+}^{-1} \mathfrak{L}$ homotopy $h: X \times[0,1] \rightarrow X_{+}$from $i$ to $g \circ f$. The Borsuk Homotopy Extension Principle [Hu, Theorem IV.2.2, p. 117], provides a homotopy $H: X_{+} \times[0,1] \rightarrow X_{+}$ such that $H_{+}=1 \mid X_{+}$and $H \circ(i \times 1 \mid[0,1])=h$. By "shortening" $H$ over $X_{+}-i(X)$, we can assume $H$ is an $f_{+}^{-1} \mathfrak{L}$-homotopy. Define the relation $g_{+}: Y_{+} \rightarrow$ $X_{+}$by $g_{+}=H_{1} \circ f_{+}^{-1} \cdot g_{+}$is actually a function because $H_{1} \circ f_{+}^{-1} \mid j(Y)=g \circ j^{-1}$ and $f_{+}^{-1} \mid Y_{+}-j(Y)$ is a function. Also $g_{+}$is continuous by A.4 because $f_{+}^{-1}$ is continuous. Thus we have a map $g_{+}: Y_{+} \rightarrow X_{+}$and an $f_{+}^{-1} \mathfrak{L}$-homotopy $H$ from $1 \mid X_{+}$to $g_{+} \circ f_{+}$.

ProOfs that (3a) implies (2a) AND that (3b) IMPlies (2b). Suppose $f_{+}: X_{+} \rightarrow Y_{+}$is a trivial extension of $f$ with associated closed embeddings $i: X \rightarrow$ $X_{+}$and $j: Y \rightarrow Y_{+}$. (In proving (3b) implies (2b), this $f_{+}$is an ANR trivial extension of $f$ which arises as follows. We are given a closed embedding $i: X \rightarrow X_{+}$into an ANR $X_{+}$, and $f_{+}: X_{+} \rightarrow Y_{+}$is the trivial extension of $f$ by $i$.) Now it suffices to prove that if $f_{+}^{-1}: Y_{+} \rightarrow X_{+}$is slice-trivial, then so is $i \circ f^{-1}: Y \rightarrow X_{+}$. Since $i \circ f^{-1}=f_{+}^{-1} \circ j$, this follows from

4.6. LEMMA. If $R: Y \rightarrow Z$ is a slice-trivial relation from a topological space $Y$ to a topological space $Z$, and $e: X \rightarrow Y$ is a proper map such that $e(X) \subset \operatorname{dom} R$, then $R \circ e: X \rightarrow Y$ is slice-trivial.

Proof. Let $U$ be a neighborhood of $R \circ e$ in $X \times Z$. Since both $e^{-1}: Y \rightarrow$ $X$ and $R: Y \rightarrow Z$ are continuous relations with compact point images, then the 
Composition Lemma A.10 provides a neighborhood $V$ of $R$ in $Y \times Z$ such that $V \circ e \subset U$. Since $R$ is slice-trivial, there is an open neighborhood $W$ of $R$ in $Y \times Z$, a map $g$ : $\operatorname{dom} W \rightarrow Z$ and a slice-contraction $\phi: W \times[0,1] \rightarrow V$ of $W$ onto $g$ in $V$. $W \circ e$ is an open neighborhood of $R \circ e$ in $X \times Z$ because $W \circ e=(e \times 1 \mid Z)^{-1}(W)$ (see A.1(3)). A slice-contraction $\psi:(W \circ e) \times[0,1] \rightarrow U$ of $W \circ e$ onto $g \circ e: X \rightarrow Z$ in $U$ is defined by the formula

$$
\psi((x, z), t)=(x, \pi \circ \phi((e(x), z), t))
$$

for $((x, z), t) \in(W \circ e) \times[0,1]$. Here $\pi: Y \times Z \rightarrow Z$ denotes projection.

PROOF THAT (2b) IMPLIES (2a) AND THAT (3b) IMPLIES (3a). These implications are immediate consequences of the following two facts. First, given the metric space $X$, there is a closed embedding $i: X \rightarrow X_{+}$into an ANR $X_{+}[\mathbf{D}$, Theorem XIII.5.2, p. 286 and Theorem IX.6.1, p. 188]. Second, given a closed embedding $i: X \rightarrow X_{+}$into an ANR $X_{+}$, the trivial extension of $f$ by $i$ is an ANR trivial extension of $f$.

PROOF THAT (2a) IMPlies (1a). Assume (2a). There is a closed embedding $i: X \rightarrow X_{+}$into a metrizable space $X_{+}$such that $i \circ f^{-1}: Y \rightarrow X_{+}$is slice-trivial. Let $\mathfrak{M}$ be an open cover of $i \circ f^{-1}$. Then Corollary A.9 provides a neighborhood $U$ of $i \circ f^{-1}$ in $Y \times X_{+}$such that $\{U(y): y \in Y\}$ refines $\mathfrak{M}$. There is a slice-contraction $\phi:\left(i \circ f^{-1}\right) \times[0,1] \rightarrow U$ of $i \circ f^{-1}$ onto a map $g: Y \rightarrow X_{+}$in $U$. Let $\pi: Y \times X_{+} \rightarrow X_{+}$ denote projection. An $\mathfrak{M}$-homotopy $h: X \times[0,1] \rightarrow X_{+}$from $i$ to $g \circ f$ is defined by the formula

$$
h(x, t)=\pi \circ \phi((f(x), i(x)), t)
$$

for $(x, t) \in X \times[0,1]$.

PROOF THAT (3b) IMPLIES (4b). This is an immediate consequence of Theorem 4.1.

ProOf THAT (4b) IMPlies (4a). The two facts that proved that (3b) implies (3a) also establish this implication.

Proof that (4a) implies (1a). Assume (4a). Let $f_{+}: X_{+} \rightarrow Y_{+}$be a trivial extension of $f$ which is a fine homotopy equivalence, and let $i: X \rightarrow X_{+}$and $j: Y \rightarrow$ $Y_{+}$be the associated closed embeddings. Let $\mathfrak{M}$ be an open cover of $i \circ f^{-1}$. Since $f_{+}^{-1}: Y_{+} \rightarrow X_{+}$is continuous, there is an open cover $\mathfrak{L}$ of $Y_{+}$such that $f_{+}^{-1} \mathfrak{L}$ refines $\mathfrak{M} \cup\left\{X_{+}-i(X)\right\}$. Since $f_{+}$is a fine homotopy equivalence, there is a map $g: Y_{+} \rightarrow X_{+}$and an $f_{+}^{-1} \mathfrak{L}_{\text {-homotopy }} h: X_{+} \times[0,1] \rightarrow X_{+}$from $1 \mid X_{+}$to $g \circ f_{+}$. It follows that $g \circ j: Y \rightarrow X_{+}$is a map and $h \circ(i \times 1 \mid[0,1]): X \times[0,1] \rightarrow X_{+}$is an $\mathfrak{M}$-homotopy from $i$ to $(g \circ j) \circ f$.

Proof that (4a) ImPlies (5). Assume (4a). Let $f_{+}: X_{+} \rightarrow Y_{+}$be a trivial extension of $f$ which is a fine homotopy equivalence, and let $i: X \rightarrow X_{+}$and $j: Y \rightarrow$ $Y_{+}$be the associated closed embeddings. Let $Z$ be an ANR. Let $B$ be a closed subset of $Y$, and let $A=f^{-1} B$. We must prove that $(f \mid A)^{\#}:[B, Z] \rightarrow[A, Z]$ is a bijection.

The following diagram is commutative.

$$
\begin{array}{ccc}
{[B, Z]} & \stackrel{(f \mid A)^{\#}}{\longrightarrow} & {[A, Z]} \\
(j \mid B)^{\# \uparrow} & & \uparrow(i \mid A)^{\#} \\
{[j(B), Z]} & \stackrel{\left(f_{+} \mid i(A)\right)^{\#}}{\longrightarrow} & {[i(A), Z]}
\end{array}
$$


$(i \mid A)^{\#}$ and $(J \mid B)^{\#}$ are bijections. Since $f_{+}$is a fine homotopy equivalence, then Lemma 4.7 implies that $\left(f_{+} \mid i(A)\right)^{\#}$ is bijective. We conclude that $(f \mid A)^{\#}$ is bijective.

4.7. LEMMA. Let $f: X \rightarrow Y$ be a proper onto map from a metrizable space $X$ to a metrizable space $Y$. If $f$ is a fine homotopy equivalence, then $f$ is a hereditary shape equivalence.

Proof. Let $Z$ be an ANR. Let $B$ be a closed subset of $Y$, and let $A=f^{-1} B$. We must prove that $(f \mid A)^{\#}:[B, Z] \rightarrow[A, Z]$ is bijective.

First, we show that $(f \mid A)^{\#}$ is surjective. Let $\phi: A \rightarrow Z$ be a map. Since $Z$ is an ANR and $f^{-1}: Y \rightarrow X$ is continuous, there is an open neighborhood $L$ of $B$ in $Y$ and a map $\psi: f^{-1} L \rightarrow Z$ such that $\psi \mid A=\phi$. Since $\mathfrak{L}=\{L, Y-B\}$ is an open cover of $Y$, there is a map $g: Y \rightarrow X$ and an $f^{-1} \mathfrak{L}$-homotopy $h: X \times[0,1] \rightarrow X$ from $1 \mid X$ to $g \circ f$. Apparently $h(A \times[0,1]) \cup g(B) \subset f^{-1} L$. So $\psi \circ g \mid B: B \rightarrow Z$ is a map, and $\psi \circ h \mid A \times[0,1]: A \times[0,1] \rightarrow Z$ is a homotopy from $\phi$ to $(\psi \circ g \mid B) \circ(f \mid A)$. Consequently, $(f \mid A)^{\#}[\psi \circ g \mid B]=[\phi]$.

Second, we prove that $(f \mid A)^{\#}$ is injective. Let $\phi_{0}, \phi_{1}: B \rightarrow Z$ be maps such that $(f \mid A)^{\#}\left[\phi_{0}\right]=(f \mid A)^{\#}\left[\phi_{1}\right]$. Then there is a homotopy $h: A \times[0,1] \rightarrow Z$ from $\phi_{0} \circ f \mid A$ to $\phi_{1} \circ f \mid A$. Since $Z$ is an ANR, there is an open neighborhood $L$ of $B$ in $Y$, there are maps $\psi_{0}, \psi_{1}: L \rightarrow Z$ such that $\psi_{0} \mid B=\phi_{0}$ and $\psi_{1} \mid B=\phi_{1}$, and there is a homotopy $H: f^{-1} L \times[0,1] \rightarrow Z$ from $\psi_{0} \circ f \mid f^{-1} L$ to $\psi_{1} \circ f \mid f^{-1} L$.

Since $\mathfrak{L}=\{L, Y-B\}$ is an open cover of $Y$, there is a map $g: Y \rightarrow X$ such that $f \circ g \sim 1 \mid Y[\mathfrak{L}]$. It follows that $f \circ g \mid B$ is homotopic to $1 \mid B$ in $L$, and that $g(B) \subset f^{-1} L$. Consequently, $H \circ(g|B \times 1|[0,1]): B \times[0,1] \rightarrow Z$ is a homotopy from $\psi_{0} \circ f \circ g \mid B$ to $\psi_{1} \circ f \circ g \mid B$. Since $f \circ g|B \sim 1| B$ in $L$, then $\psi_{t} \circ f \circ g\left|B \sim \psi_{t}\right| B=\phi_{t}$ for $t=0$ or 1 . By stringing together the homotopies

$$
\phi_{0} \sim \psi_{0} \circ f \circ g\left|B \sim \psi_{1} \circ f \circ g\right| B \sim \phi_{1},
$$

we conclude that $\left[\phi_{0}\right]=\left[\phi_{1}\right]$.

Proof that (5) Implies (1b). Assume (5). Let $\phi: X \rightarrow Z$ be a map into an ANR $Z$, and let $\mathfrak{M}$ be an open cover of $\phi \circ f^{-1}: Y \rightarrow Z$. Here we shall invoke two fundamental results of $[\mathbf{K 2}]$ concerning hereditary shape equivalences. We shall apply Lemmas 5 and 6 of [K2], substituting our $f: X \rightarrow Y$ and $\phi: X \rightarrow Z$ for Kozlowski's $f: X^{\prime} \rightarrow X$ and $h: X^{\prime} \rightarrow Y$, respectively. Lemma 5 implies that the extension condition in the hypothesis of Lemma 6 is satisfied. To apply Lemma 6 , let $\mathfrak{C}$ be a locally finite closed cover of $Y$ such that $\left\{\phi \circ f^{-1}(C): C \in \mathfrak{C}\right\}$ refines $\mathfrak{M}$, and let $O: \mathfrak{C} \rightarrow \mathfrak{M}$ be a function such that $\phi \circ f^{-1}(C) \subset O(C)$ for each $C \in \mathfrak{C}$. Then Lemma 6 provides an $\mathfrak{M}$-homotopy $H: X \times[0,1] \rightarrow Z$ such that $H_{0}=\phi$ and $H_{1}=g \circ f$ for some map $g: Y \rightarrow Z$.

We remark that this proof is a minor variation of the proof of Theorem 9 of [K2].

This completes the proof of Theorem 4.5.

The following corollary is a result of Kozlowski (Theorem 9 of [K2]). We repeat it here in order to give two alternatives to Kozlowski's proof. The first alternative illustrates an interesting relation-theoretic technique. The second alternative is very short. 
4.8. Corollary. Let $f: X \rightarrow Y$ be a proper onto map from an ANR $X$ to a metrizable space $Y$. If $f$ is a hereditary shape equivalence, then $Y$ is an ANR.

FIRST PROOF. Let $i: Y \rightarrow Z$ be a closed embedding into a metrizable space $Z$. We shall find a neighborhood $N$ of $i(Y)$ in $Z$ and a map $r: N \rightarrow Y$ such that $r \circ i=1 \mid Y$.

Since $f$ is an ANR trivial extension of itself, Theorem 4.5 implies that $f^{-1}: Y \rightarrow$ $X$ is slice-trivial. We assert that $(i \circ f)^{-1}: Z \rightarrow X$ is slice-trivial. This assertion will follow from Lemma 4.3 once we show that $(i \circ f)^{-1}$ is slice-contractible in each of its neighborhoods in $Z \times X$. To this end, let $U$ be a neighborhood of $(i \circ f)^{-1}$ in $Z \times X$. Then $(i \times 1 \mid X)^{-1}(U)$ is a neighborhood of $f^{-1}$ in $Y \times X$, and there is a slice-contraction $\phi: f^{-1} \times[0,1] \rightarrow(i \times 1 \mid X)^{-1}(U)$ of $f^{-1}$ in $(i \times 1 \mid X)^{-1}(U)$. Since $(i \times 1 \mid X)^{-1}\left((i \circ f)^{-1}\right)=f^{-1}$ (using A.1), then

$$
(i \times 1 \mid X) \circ \phi \circ\left((i \times 1 \mid X)^{-1} \times 1 \mid[0,1]\right) \mid(i \circ f)^{-1} \times[0,1]
$$

is a slice-contraction of $(i \circ f)^{-1}$ in $U$. Our assertion follows.

Since $(i \circ f)^{-1}: Z \rightarrow X$ is slice-trivial and $\operatorname{dom}(i \circ f)^{-1}=i(Y)$, then Proposition 2.4 (The Neighborhood Extension Property) provides a continuous relation $S: Z \rightarrow$ $Y$ with the following properties. If $N=\operatorname{dom} S$, then $N$ is a neighborhood of $i(Y)$ in $Z . S \mid i(Y)=(i \circ f)^{-1}$ and $S \mid N-i(Y)$ is a function. Define the relation $g: N \rightarrow Y$ by $g=f \circ S$. The continuity of $g$ is guaranteed by A.4. In fact, $g$ is actually a function, because both $g \mid i(Y)=i^{-1}$ and $S \mid N-i(Y)$ are functions. Since $g \circ i=1 \mid Y$, we are done.

SECOND PROOF. Since $f$ is an ANR trivial extension of itself, Theorem 4.5 implies that $f$ is a fine homotopy equivalence. Thus for every open cover $\mathfrak{L}$ of $Y$, there is a map $g: Y \rightarrow X$ such that $f \circ g \sim 1 \mid Y[\mathfrak{L}]$. Since $X$ is an ANR, then according to Theorem IV.6.3 on pp. 139-140 of [Hu], $Y$ must be an ANR.

We conclude this section by exploring two questions which are provoked by Theorem 4.1 and Corollary 4.4. Let $f: X \rightarrow Y$ be a proper onto map from a metrizable space $X$ to a metrizable space $Y$. If $f$ is a fine homotopy equivalence, is $f^{-1}: Y \rightarrow X$ necessarily slice-trivial? If $f$ is a fine homotopy injection, is it necessarily a fine homotopy equivalence? Corollary 4.4 tells us that the answer to both these questions is "yes" whenever $X$ is an ANR. However, if $X$ is not an ANR, the answer to the first question can be "no" (as we shall illustrate below) and the answer to the second question is not known.

The following definition aids in the investigation of the first question. Proposition 3.1 implies that a topological space $Y$ is locally contractible if $1 \mid Y$ is slice-trivial in $Y \times Y$. This motivates us to define a topological space $Y$ to be uniformly locally contractible if $1 \mid Y$ is slice-trivial in $Y \times Y$.

The identity map of any topological space is a fine homotopy equivalence. Thus a special case of the first of our two questions is the following. Is a metrizable space necessarily uniformly locally contractible? The answer, of course, is "no". Indeed, if $Y$ is a metrizable space which is not locally contractible (such as the subspace $\left\{0, \frac{1}{2}, \frac{1}{3}, \frac{1}{4}, \ldots\right\}$ of the real line), then $Y$ is certainly not uniformly locally contractible. In other words, if a metrizable space $Y$ is not a locally contractible, then $1 \mid Y$ is a fine homotopy equivalence whose inverse is not slice-trivial.

A modification of the preceding question which is perhaps less trivial and more interesting is the following. If a metrizable space is locally contractible, is it nec- 
essarily uniformly locally contractible? Again the answer is "no". To see this, we take $\mathfrak{B}$ to be Borsuk's locally contractible compact metrizable space which is not an ANR [Hu, pp. 169-172]. Inspection of $\mathfrak{B}$ reveals that each neighborhood of $1 \mid \mathfrak{B}$ in $\mathfrak{B} \times \mathfrak{B}$ contains a map from $\mathfrak{B}$ to itself which is not homotopic to $1 \mid \mathfrak{B}$. (Below we give a rough description of $\mathfrak{B}$ to suggest where these maps come from.) It follows via Proposition 2.3 (Homotopy Uniqueness), that $1 \mid \mathfrak{B}$ is not slice-trivial. Hence $\mathfrak{B}$ is not uniformly locally connected.

Here is the rough description of $\mathfrak{B}$ promised above. $\mathfrak{B}=\left(\bigcup_{n=1}^{\infty} S_{n}\right) \cup K$ where each $S_{n}$ is an $n$-dimensional sphere and $K$ is a Hilbert cube. Furthermore for each $n \geq 1, S_{n} \cap S_{n+1}=K_{n}$ is an $n$-cell, and $S_{n} \cap\left(\left(\bigcup_{k=n+2}^{\infty} S_{k}\right) \cup K\right)=\emptyset$. For each $n \geq 1$ we can find a retraction map $r_{n}: \mathfrak{B} \rightarrow \bigcup_{k=1}^{n} S_{k}$ such that

$$
r_{n}\left(\left(\bigcup_{k=n+1}^{\infty} S_{k}\right) \cup K\right)=K_{n}
$$

and such that the sequence $\left\{r_{n}\right\}$ converges uniformly to $1 \mid \mathfrak{B}$. The inclusion $i_{n+1}$ : $S_{n+1} \rightarrow \mathfrak{B}$ is not homotopic to a constant map. However, since $r_{n}$ 。 $i_{n+1}\left(S_{n+1}\right) \subset K_{n}$, it follows that $r_{n} \circ i_{n+1}: S_{n+1} \rightarrow \mathfrak{B}$ is homotopic to a constant map. Therefore $r_{n}$ is not homotopic to $1 \mid \mathfrak{B}$.

Borsuk's space $\mathfrak{B}$ is not uniformly locally contractible, but every ANR is uniformly locally contractible (see Corollary 4.11). The preceding observations lead us to replace the first of our two original questions by the following less trivial and more interesting query.

4.9. Question. If a compact metrizable space is uniformly locally contractible, is it necessarily an ANR?

The following result may shed some light on Question 4.9.

4.10. Proposition. If $Y$ is a metrizable space, then the following three statements are equivalent.

(1) $Y$ is uniformly locally contractible.

(2) For every open cover $\mathfrak{L}$ of $Y$, there is an open cover $\mathfrak{M}$ of $Y$ with the following property. If $f, g: X \rightarrow Y$ are maps from a topological space $X$ to $Y$ such that $f$ is within $\mathfrak{M}$ of $g$, then $f$ is $\mathfrak{L}$-homotopic to $g$.

(3) If $f: X \rightarrow Y$ is a proper map from a metrizable space $X$ to $Y$, then $f$ is slice-trivial in $X \times Y$.

Proof that (1) IMPlies (2). Assume (1). Let $\mathfrak{L}$ be an open cover of $Y$. A.9 provides a neighborhood $U$ of $1 \mid Y$ in $Y \times Y$ such that $\{U(y): y \in Y\}$ refines $\mathfrak{L}$. Then there is a neighborhood $V$ of $1 \mid Y$ in $Y \times Y$ and a slice-contraction $\phi: V \times[0,1] \rightarrow U$ of $V$ in $U$. Let $\mathfrak{M}$ be an open cover of $Y$ such that $M \times M \subset V$ for each $M \in \mathfrak{M}$. A slice-contraction $\psi: V \times[0,1] \rightarrow U$ of $V$ onto $1 \mid Y$ in $U$ can be defined as follows.

$$
\psi((y, z), t)= \begin{cases}\phi((y, z), 2 t) & \text { for }((y, z), t) \in V \times\left[0, \frac{1}{2}\right] \\ \phi((y, y), 2-2 t) & \text { for }((y, z), t) \in V \times\left[\frac{1}{2}, 1\right]\end{cases}
$$

Now suppose $f, g: X \rightarrow Y$ are maps from a topological space $X$ to $Y$, such that $f$ is within $\mathfrak{M}$ of $g$. Then $\{(g(x), f(x)): x \in X\} \subset V$. Hence a homotopy $h: X \times[0,1] \rightarrow Y$ from $f$ to $g$ is defined by the formula

$$
h(x, t)=\pi \circ \phi((g(x), f(x)), t)
$$


for $(x, t) \in X \times[0,1]$. Here $\pi: Y \times Y \rightarrow Y$ denotes projection onto the second coordinate: $\pi(y, z)=z$ for $(y, z) \in Y \times Y$. Since $h(\{x\} \times[0,1]) \subset U(g(x))$ for each $x \in X$, then $h$ is an $\mathfrak{L}$-homotopy from $f$ to $g$.

PROOF ThAT (2) Implies (3). Assume (2). Let $f: X \rightarrow Y$ be a proper map from a metrizable space $X$ to $Y$. Let $U$ be a neighborhood of $f$ in $X \times Y$. Since $f^{-1}: Y \rightarrow X$ is continuous with compact point images, there is an open cover $\mathfrak{L}$ of $Y$ such that $f^{-1} L \times L \subset U$ for each $L \in \mathfrak{L}$. Now (2) provides an open cover $\mathfrak{M}$ of $Y$ with the property that if $g, h: Z \rightarrow Y$ are maps from a topological space $Z$ to $Y$ such that $g$ is within $\mathfrak{M}$ of $h$, then $g \sim h[\mathfrak{L}]$. Next invoke A.9 to obtain a neighborhood $V$ of $f$ in $X \times Y$ such that $\{V(x): x \in X\}$ refines $\mathfrak{M}$.

Define the maps $h_{0}, h_{1}: V \rightarrow Y$ by $h_{0}(x, y)=y$ and $h_{1}(x, y)=f(x)$ for $(x, y) \in$ $V$. Then $h_{0}$ is within $\mathfrak{M}$ of $h_{1}$. Hence there is an $\mathfrak{L}$-homotopy $h: V \times[0,1] \rightarrow Y$ from $h_{0}$ to $h_{1}$. Now a slice-contraction $\phi: V \times[0,1] \rightarrow X \times Y$ of $V$ onto $f$ in $X \times Y$ is defined by the formula

$$
\phi((x, y), t)=(x, h((x, y), t))
$$

for each $((x, y), t) \in V \times[0,1]$. Since $h$ is an $\mathfrak{L}$-homotopy, it is easy to see that for each $(x, y) \in V$, there is an $L \in \mathfrak{L}$ such that $\phi(\{(x, y)\} \times[0,1]) \subset f^{-1} L \times L$. Thus $\phi$ is a slice-contraction of $V$ onto $f$ in $U$.

PROOF THAT (3) IMPLIES (1). This is obvious.

According to [Hu, Theorem IV.1.1, p. 111], every ANR satisfies condition (2) of Proposition 4.11. This gives us two corollaries.

4.11. COROLlARY. Every ANR is uniformly locally contractible.

4.12. COROLlaRY. If $f: X \rightarrow Y$ is a proper map from a metrizable space $X$ to an ANR $Y$, then $f$ is slice-trivial in $X \times Y$.

An approximate $A N R$ is a metrizable space $X$ with the property that for every open cover $\mathfrak{L}$ of $X$, there is an ANR $Y$ and maps $f: X \rightarrow Y$ and $g: Y \rightarrow X$ such that $g \circ f$ is within $\mathfrak{L}$ of $1 \mid X$.

4.13. COROLlARY. A uniformly locally contractible approximate ANR is an $A N R$.

ProOF. Suppose $X$ is a uniformly locally contractible approximate ANR. Using Proposition 4.10, we see that for every open cover $\mathfrak{L}$ of $X$, there is an ANR $Y$ and maps $f: X \rightarrow Y$ and $g: Y \rightarrow X$ such that $g \circ f \sim 1 \mid X[\mathfrak{L}]$. According to Theorem IV.6.3 on pp. $139-140$ of $[\mathbf{H u}], X$ must be an ANR.

In light of Corollary 4.13, we can rephrase Question 4.9 as follows. If a metrizable space is uniformly locally contractible, is it necessarily an approximate ANR?

We observe that Borsuk's space $\mathfrak{B}$ mentioned above is an approximate ANR. We can see this in terms of our rough description of $\mathfrak{B}$. For each $n \geq 1, \mathfrak{B}_{n}=\bigcup_{k=1}^{n} S_{k}$ is a compact polyhedron and, thus, an ANR. Also for each $n \geq 1$, we have maps $r_{n}: \mathfrak{B} \rightarrow \mathfrak{B}_{n}$ and the inclusion $j_{n}: \mathfrak{B}_{n} \rightarrow \mathfrak{B}$ such that the sequence $\left\{j_{n} \circ r_{n}\right\}$ converges uniformly to $1 \mid \mathfrak{B}$. It follows that $\mathfrak{B}$ is an approximate ANR.

We now consider the second of our two original questions. We give it official status.

4.14. Question. If a proper onto map between metrizable spaces is a fine homotopy injection, is it necessarily a fine homotopy equivalence? 
As we noted earlier, Corollary 4.4 tells us that the answer is "yes" if the domain is an ANR. Another relevant observation is the following.

4.15. COROllaRY. Let $f: X \rightarrow Y$ be a proper onto map between metrizable spaces. If $f$ is a fine homotopy injection and $Y$ is uniformly locally contractible, then $f$ is a fine homotopy equivalence.

PROOF. Let $\mathfrak{L}$ be an open cover of $Y$. Proposition 4.10 provides an open cover $\mathfrak{M}$ of $Y$ such that any map from $Y$ to itself which is within $\mathfrak{M}$ of $1 \mid Y$ is $\mathfrak{L}$ homotopic to $1 \mid Y$. There is a map $g: Y \rightarrow X$ such that $g \circ f \sim 1 \mid X\left[f^{-1} \mathfrak{M}\right]$. It follows that $f \circ g$ is within $\mathfrak{M}$ of $1 \mid Y$. Hence $f: g \sim 1 \mid Y[\mathfrak{L}]$.

Thus Question 4.14 is of interest only when restricted to a map whose domain is not an ANR and whose range is not uniformly locally contractible.

We end this section by using Theorem 4.5 to answer a weak form of Question 4.14.

4.16. COROLlaRY. Let $f: X \rightarrow Y$ be a proper onto map between metrizable spaces. If $f$ is a fine homotopy injection, then $f$ is a hereditary shape equivalence.

Proof. It suffices to verify condition (1a) of Theorem 4.5. Set $X_{+}=X$ and $i=1 \mid X$. Let $\mathfrak{M}$ be an open cover of $f^{-1}: Y \rightarrow X$. Since $f$ is proper, there is an open cover $\mathfrak{L}$ of $Y$ such that $f^{-1} \mathfrak{L}$ refines $\mathfrak{M}$. There is a map $g: Y \rightarrow X$ such that $g \circ f \sim 1 \mid X\left[f^{-1} \mathfrak{L}\right]$. Hence $g \circ f \sim i[\mathfrak{M}]$.

5. Applications of the Main Theorem. This section contains three applications of Main Theorem 3.2 together with a number of corollaries.

The first application, Theorem 5.1, formulates a property which forces a cell-like map to be a hereditary shape equivalence. From this, in Corollary 5.2, we extract a condition on the singular set of a cell-like map which suffices to make the map a hereditary shape equivalence. We study this singular set condition in order to draw applications from Corollary 5.2. We first discuss why a naturally conjectured and highly optimistic application of Corollary 5.2 fails to work. We are then drawn into some dimension-theoretic considerations, in 5.4 and 5.5 , in order to deduce a more modest application of Corollary 5.2 which includes a theorem of Kozlowski; see Corollary 5.6. Corollaries 5.7 and 5.8 are immediate consequences of Theorem 5.1 ; they state that if the restrictions of a cell-like map to certain closed subsets are hereditary shape equivalences, then so is the cell-like map itself.

The second application of Main Theorem 3.2 is Theorem 5.9 which derives certain conclusions about the image space of a $U V^{\infty}$ map. These conclusions are particularly interesting when specialized to the identity map of a locally contractible space, as is done in Corollary 5.10. The proof of 5.9 stimulates an observation about spaces which are absolute neighborhood extensors for the class of countable dimensional metrizable spaces; this observation is verified in 5.11 and 5.12. These results give rise to several questions which are recorded in 5.13.

The third application of the Main Theorem, to be found in 5.14, is a new proof of Haver's theorem [H1] that a cell-like map between ANR's is a fine homotopy equivalence, without the separability hypothesis required in $[\mathbf{H 1}]$.

5.1. THEOREM. A cell-like map $f: X \rightarrow Y$ from a metrizable space $X$ to a metrizable space $Y$ is a hereditary shape equivalence if there is a sequence $\left\{K_{n}\right\}$ of 
closed subsets of $Y$ such that:

(1) $Y-\bigcup_{n=1}^{\infty} K_{n}$ is countable dimensional, and

(2) $f \mid f^{-1}\left(K_{n}\right): f^{-1}\left(K_{n}\right) \rightarrow K_{n}$ is a hereditary shape equivalence for each $n \geq 1$.

PROOF. There is a closed embedding $i: X \rightarrow X_{+}$into an ANR $X_{+}$. (Use [D, Theorem XIII.5.2, p. 286 and Theorem IX.6.1, p. 188].) According to Theorem 4.5, it suffices to prove that $i \circ f^{-1}: Y \rightarrow X_{+}$is slice-trivial. We shall do this by means of Main Theorem 3.2. To satisfy the hypotheses of Theorem 3.2, we must show that $i \circ f^{-1} \mid K_{n}$ is slice-trivial in $Y \times X_{+}$for each $n \geq 1$.

Let $n \geq 1$. Theorem 4.5 implies that $i \circ f^{-1} \mid K_{n}: K_{n} \rightarrow X_{+}$is slice-trivial in $K_{n} \times X_{+}$. Therefore $i \circ f^{-1} \mid K_{n}$ is slice-contractible in each of its neighborhoods in $Y \times X_{+}$. Since $X_{+}$is an ANR, Lemma 4.3 implies that $i \circ f^{-1} \mid K_{n}$ is slicetrivial in $Y \times X_{+}$.

The corollaries to Theorem 5.1 use the following terminology. Let $f: X \rightarrow Y$ be a function. We define the singular set of $f$, denoted $S(f)$, to be $\left\{y \in Y: f^{-1}(y)\right.$ contains more than one point\}.

The first corollary to Theorem 5.1 generalizes several results of Kozlowski (Theorems 12, 13 and Corollaries $5-8$ of $[\mathbf{K 2}]$.)

5.2. Corollary. A cell-like map between metrizable spaces is a hereditary shape equivalence if its singular set is contained in a countable dimensional $G_{\delta}$ subset of its range.

ProOF. Let $f: X \rightarrow Y$ be a cell-like map between metrizable spaces whose singular set is contained in a countable dimensional $G_{\delta}$ subset $G$ of $Y$. Then $G=$ $Y-\bigcup_{n=1}^{\infty} K_{n}$ where each $K_{n}$ is a closed subset of $Y$, and $f \mid f^{-1} K_{n}: f^{-1} K_{n} \rightarrow K_{n}$ is a homeomorphism for each $n \geq 1$. Since a homeomorphism is a hereditary shape equivalence, the hypotheses of Theorem 5.1 are satisfied.

5.3. COROLlary. A cell-like map between metrizable spaces is a hereditary shape equivalence if its range is countable dimensional.

Corollary 5.2 suggests inquiring into conditions which will guarantee that a subset of a metrizable space is contained in a countable dimensional $G_{\delta}$ subset. One such condition is provided by a theorem of L. Tumarkin which says that a finite dimensional subset of a metrizable space is contained in a $G_{\delta}$ subset of the same dimension [N, Theorem II.10, p. 32]). Thus, a cell-like map between metrizable spaces is a hereditary shape equivalence if its singular set is finite dimensional.

It is natural, if optimistic, to conjecture a countable dimensional version of Tumarkin's theorem: a countable dimensional subset of a metrizable space is contained in a countable dimensional $G_{\delta}$ subset. If true, this conjecture would yield the following strenthened version of Corollary 5.2: a cell-like map between metrizable spaces is a hereditary shape equivalence if its singular set is countable dimensional. Unfortunately, both the countable dimensional version of Tumarkin's theorem and the strengthened version of Corollary 5.2 are false. We shall now comment on the failure of these two conjectures in more detail.

Tumarkin's theorem can be proved by first establishing it for zero-dimensional subsets and then deducing the $n$-dimensional case $(0<n<\infty)$ from the following two facts. (1) The dimension of a metrizable space is $\leq n$ if and only if the space 
is the union of $\leq n+1$ zero-dimensional subsets. (2) The union of a finite number of $G_{\delta}$ subsets is a $G_{\delta}$ subset. Unfortunately, this technique does not establish the countable dimensional case, because the union of a countable number of $G_{\delta}$ subsets need not be a $G_{\delta}$ subset.

We now give a specific illustration of the failure of the countable dimensional version of Tumarkin's theorem. For any topological space $X$, let $X^{\infty}=X \times$ $X \times X \times \cdots$. The Hilbert cube is the space $I^{\infty}=[0,1]^{\infty}$. For each $n \geq 1$, let $I^{n}=[0,1]^{n} \times\{0\}^{\infty}$; and let $S=\bigcup_{n=1}^{\infty} I^{n} . S$ is a countable dimensional subset of $I^{\infty}$. We shall argue that any $G_{\delta}$ subset of $I^{\infty}$ which contains $S$ also contains a Hilbert cube. Since the Hilbert cube is not countable dimensional $[\mathbf{N}$, Theorem VI.1.B, p. 164], it will follow that $S$ is not contained in any countable dimensional $G_{\delta}$ subset of $I^{\infty}$. Suppose $S \subset \bigcap_{n=1}^{\infty} O_{n}$ where each $O_{n}$ is an open subset of $I^{\infty}$. Then one can inductively select a decreasing sequence of positive numbers $\left\{\varepsilon_{n}\right\}$ such that $I^{n-1} \times\left[0, \varepsilon_{n}\right]^{\infty} \subset O_{n}$ for each $n \geq 1$. It follows that $\bigcap_{n=1}^{\infty} O_{n}$ contains the Hilbert cube $\left[0, \varepsilon_{1}\right] \times\left[0, \varepsilon_{2}\right] \times\left[0, \varepsilon_{3}\right] \times \cdots$.

Not only is the countable dimensional version of Tumarkin's theorem false. As we mentioned earlier, the conjectured strengthening of Corollary 5.2 is itself false. In [DW], R. J. Daverman and J. J. Walsh modify J. Taylor's construction in [T] to produce a cell-like map from a compact metrizable space to the Hilbert cube which has a countable dimensional singular set but which is not a hereditary shape equivalence. Corollary 5.2 tells us that the singular set of this map is another instance of a countable dimensional subset of the Hilbert cube which is not contained in a countable dimensional $G_{\delta}$ subset.

Although the most optimistic conjecture concerning Tumarkin's theorem (the countable dimensional version) fails; nevertheless, there is a more modest strengthening of Tumarkin's theorem (the locally finite dimensional version) which can be proved. To this end, we define a metrizable space to be locally finite dimensional if each of its points has a finite dimensional neighborhood. We observe that a locally finite dimensional space is countable dimensional. Indeed, if $L$ is locally finite dimensional, then $L=\bigcup_{n=0}^{\infty} L_{n}$ where

$$
L_{n}=\bigcup\{O: O \text { is an open subset of } L \text { and } \operatorname{dim} O \leq n\}
$$

for each $n \geq 0$. Since $L_{n}$ is paracompact, it can be covered by a locally finite collection of closed subsets of dimension $\leq n$. Now Theorem II.2.D on p. 16 of [N] tells us that $\operatorname{dim} L_{n} \leq n$ for each $n \geq 0$. We conclude that $L$ is countable dimensional.

5.4. THE LOCALly FINITE DIMENSIONAL VERSION OF TUMARKIN'S THEOREM. A locally finite dimensional subset of a metrizable space is contained in a locally finite dimensional $G_{\delta}$ subset.

PROOF. Let $L$ be a locally finite dimensional subset of a metrizable space $X$. For each $n \geq 0$, define $L_{n}$ as in the paragraph preceding the statement of 5.4. Then $L=\bigcup_{n=0}^{\infty} L_{n}$; and for each $n \geq 0, L_{n}$ is a (relatively) open subset of $L, L_{n} \subset L_{n+1}$ and $\operatorname{dim} L_{n} \leq n$.

For each $n \geq 0$, let $M_{n}$ be an open subset of $X$ such that $L \cap M_{n}=L_{n}$. We can assume $M_{n} \subset M_{n+1}$ for each $n \geq 0$; for since $L_{i} \subset L_{i+1}$ for each $i \geq 0$, then $M_{n}$ can be replaced by $\bigcup_{i=0}^{n} M_{i}$. 
For each $n \geq 0$, Tumarkin's theorem [N, Theorem II.10, p. 32] provides a finite dimensional $G_{\delta}$ subset $G_{n}$ of $X$ such that $L_{n} \subset G_{n}$. We can assume $G_{n} \subset G_{n+1}$ for each $n \geq 0$; for since the union of a finite number of finite dimensional $G_{\delta}$ subsets is a finite dimensional $G_{\delta}$ subset, then $G_{n}$ can be replaced by $\bigcup_{i=0}^{n} G_{i}$.

Let $L_{-1}=M_{-1}=\emptyset$, and define $H=\bigcup_{n=0}^{\infty}\left(M_{n}-M_{n-1}\right) \cap G_{n}$. Then $L \subset H$, because $L_{n}-L_{n-1} \subset\left(M_{n}-M_{n-1}\right) \cap G_{n}$ for each $n \geq 0$, and $L=\bigcup_{n=0}^{\infty}\left(L_{n}-L_{n-1}\right)$. $H$ is locally finite dimensional because $H \cap M_{n} \subset G_{n}$ for each $n \geq 0$.

We now argue that $H$ is a $G_{\delta}$ subset of $X$. Let $M=\bigcup_{n=0}^{\infty} M_{n}$. Since $M_{n-1} \subset$ $M_{n}$ for each $n \geq 0$, then $M$ is the disjoint union of the sets

$$
\left(M_{n}-M_{n-1}\right) \cap G_{n}, \quad\left(M_{n}-M_{n-1}\right)-G_{n}, \quad n \geq 0 .
$$

Thus $H=M-\bigcup_{n=0}^{\infty}\left(\left(M_{n}-M_{n-1}\right)-G_{n}\right)$. For each $n \geq 0,\left(M_{n}-M_{n-1}\right)-G_{n}=$ $M_{n} \cap\left(X-M_{n-1}\right) \cap\left(X-G_{n}\right)$. Since $M_{n}, X-M_{n-1}$ and $X-G_{n}$ are $F_{\sigma}$ subsets, and since the intersection of a finite number of $F_{\sigma}$ subsets is an $F_{\sigma}$ subset, then $\left(M_{n}-M_{n-1}\right)-G_{n}$ is an $F_{\sigma}$ subset for each $n \geq 0$. Hence $\bigcup_{n=0}^{\infty}\left(\left(M_{n}-M_{n-1}\right)-G_{n}\right)$ is an $F_{\sigma}$ subset. Since $M$ is an open set, it follows that $H$ is a $G_{\delta}$ subset.

With the aid of 5.4, we now observe that if $S$ is a subset of a metrizable space $X$, and all but a locally finite dimensional subset of $S$ is contained in a countable dimensional $G_{\delta}$ subset $G$ of $X$, then $S$ is totally contained in a countable dimensional $G_{\delta}$ subset of $X$. For 5.4 implies that $S-G$ is contained in a locally finite dimensional $G_{\delta}$ subset $M$ of $X$. As we observed earlier, $M$ must be countable dimensional. So $G \cup M$ is a countable dimensional $G_{\delta}$ set which contains all of $S$.

The preceding observation yields the following information. (See $[\mathbf{N}$, p. 161], for the definition of strong transfinite dimension.)

5.5. COROLLARY. If a subset $S$ of a metrizable space $X$ has strong transfinite dimension, then $S$ lies in a countable dimensional $G_{\delta}$ subset of $X$.

Proof. We invoke Theorem VI.3.13, p. 178, the Corollary to Theorem VI.2, p. 168, and Theorem VI.6, p. 178 of $[\mathbf{N}]$ in order to write $S=K \cup L$ where $K$ is compact and $L$ is locally finite dimensional. $K$ has strong transfinite dimension because it is a closed subset of $S$. (This is proved for strong finite inductive dimension in II.1.A, p. 11 of $[\mathbf{N}]$. Essentially the same proof works for strong transfinite dimension.) Hence $K$ is countable dimensional [N, Theorem VI.3,B, p. 178]. Also $K$ is a $G_{\delta}$ subset because it is compact. The observation following 5.4 now finishes the proof.

Combining Corollary 5.2, Proposition 5.4, the observation following 5.4 and Corollary 5.5 , we have

5.6. COROLlaRY. A cell-like map between metrizable spaces is a hereditary shape equivalence if its singular set satisfies any of the following conditions.

(1) The singular set is locally finite dimensional.

(2) All but a locally finite dimensional subset of the singular set is contained in a countable dimensional $G_{\delta}$ subset of the range.

(3) The singular set is contained in a subset of the range which has strong transfinite dimension.

Corollary 5.6(3) is Theorem 12 of [K2].

We extract another corollary from Theorem 5.1. Like Corollary 5.2, it generalizes Corollary 8 of $[\mathbf{K 2}]$, but in a different direction. 
5.7. Corollary. A cell-like map $f: X \rightarrow Y$ from a metrizable space $X$ to a metrizable space $Y$ is a hereditary shape equivalence if there is a sequence $\left\{K_{n}\right\}$ of closed subsets of $Y$ such that $Y=\bigcup_{n=1}^{\infty} K_{n}$ and $f \mid f^{-1}\left(K_{n}\right): f^{-1}\left(K_{n}\right) \rightarrow K_{n}$ is a hereditary shape equivalence for each $n \geq 1$.

The proof is immediate from Theorem 5.1.

The next corollary is just Lemma 8 of $[\mathbf{K 2}]$. We include it in order to exhibit a very swift proof of it from Corollary 5.7.

5.8. Corollary. A cell-like map $f: X \rightarrow Y$ from a metrizable space $X$ to a metrizable space $Y$ is a hereditary shape equivalence if there is a closed subset $K$ of $Y$ such that $S(f) \subset K$ and $f \mid f^{-1}(K): f^{-1}(K) \rightarrow K$ is a hereditary shape equivalence.

ProOF. The open set $Y-K$ is the union of a sequence $\left\{L_{n}\right\}$ of closed subsets of $Y$, and $f \mid f^{-1}\left(L_{n}\right): f^{-1}\left(L_{n}\right) \rightarrow L_{n}$ is a homeomorphism for each $n \geq 1$. Since a homeomorphism is a hereditary shape equivalence, the hypotheses of Corollary 5.7 are satisfied.

We now turn to our second application of the Main Theorem.

5.9. THEOREM. Let $f: X \rightarrow Y$ be a $U V^{\infty}$ map from a metrizable space $X$ to a metrizable space $Y$. Then $Y$ is an ANE for the class of all countable dimensional metrizable spaces. Furthermore, if $Y$ is countable dimensional, then $Y$ is an ANR.

Before giving the proof of this theorem, we state a corollary. Since the identity map of a locally contractible space is a $U V^{\infty}$ map, then Theorem 5.9 implies

5.10. COROLLARY. Every locally contractible metrizable space is an ANE for the class of all countable dimensional metrizable spaces. Furthermore, every locally contractible countable dimensional metrizable space is an ANR.

This corollary was proved independently and earlier via different techniques by D. F. Addis and H. J. Gresham in [AG and $\mathbf{G}]$.

PROOF OF THEOREM 5.9. We assert that $Y$ has the following property. If $A$ is a countable dimensional closed subset of a metrizable space $Z$, then any map $\phi: A \rightarrow Y$ extends to a map $\psi: N \rightarrow Y$ where $N$ is a neighborhood of $A$ in $Z$.

Let $\phi: A \rightarrow Y$ be a map. Main Theorem 3.2 implies that $f^{-1} \circ \phi$ is slice-trivial in $Z \times X$ (not just in $A \times X)$. Since $\operatorname{dom}\left(f^{-1} \circ \phi\right)=A$, then Proposition 2.4 (The Neighborhood Extension Property) provides a continuous relation $S: Z \rightarrow X$ with the following properties. If $N=\operatorname{dom} S$, then $N$ is a neighborhood of $A$ in $Z$, $S \mid A=f^{-1} \circ \phi$ and $S \mid N-A$ is a function. Define the relation $\psi: N \rightarrow Y$ by $\psi=f \circ S$. The continuity of $\psi$ is guaranteed by A.4. Moreover, $\psi$ is a function which extends $\phi$, because both $\psi \mid A=\phi$ and $S \mid N-A$ are functions. This proves our assertion.

From the assertion it follows immediately that $Y$ is an ANE for the class of all countable dimensional metrizable spaces.

Now assume $Y$ is countable dimensional. Let $i: Y \rightarrow Z$ be a closed embedding into a metrizable space $Z$. The assertion implies that $i^{-1}: i(Y) \rightarrow Y$ extends to a map $r: N \rightarrow Y$ where $N$ is a neighborhood of $i(Y)$ in $Z$. Hence $r \circ i=1 \mid Y$. We conclude that $Y$ is an ANR. 
The assertion verified at the beginning of the preceding proof endows $Y$ with a property that is apparently stronger than being an ANE for the class of countable dimensional metrizable spaces. In fact, these two properties are equivalent. We shall sketch a proof of this equivalence.

First one establishes

5.11. LemMA. Let $A$ be a closed subset of a metrizable space $X$. Then there is a metrizable space $Z$ and a map $g: X \rightarrow Z$ such that $g \mid A: A \rightarrow Z$ is a closed embedding and $Z-g(A)$ is countable dimensional.

ProOF. Roughly speaking, $Z$ is the disjoint union of $A$ and $|n(\mathfrak{L})|$ where $\mathfrak{L}$ is an open cover of $X-A, n(\mathfrak{L})$ is the nerve of $\mathfrak{L}$, and $|n(\mathfrak{L})|$ is the polyhedron underlying $n(\mathfrak{L})$ with the metric topology. The map $g: X \rightarrow Z$ consists of two "pieces": $g|A=1| A$, and $g \mid X-A$ is a barycentric map from $X-A$ to $|n(\mathfrak{L})|$.

Here are the details. Let $\rho$ be a bounded metric on $X$. Let $\mathfrak{L}$ be an open cover of $X-A$ such that $\operatorname{diam} L<\rho(L, A)$ for each $L \in \mathfrak{L}$. $n(\mathfrak{L})$ denotes the nerve of $\mathfrak{L}$, and $|n(\mathfrak{L})|$ denotes the polyhedron underlying $n(\mathfrak{L})$ equipped with the metric topology (see Appendix B). Let $\beta:(X-A) \rightarrow|n(\mathfrak{L})|$ be a barycentric map; thus for any $x \in X-A$ and any $L \in \mathfrak{L}, \beta(x)(L) \neq 0$ implies $x \in L$ (see Proposition B.1).

Theorem XIII.5.2, p. 286 of [D] provides an isometric embedding $i: X \rightarrow C(X)$ where $C(X)$ denotes the Banach space of all bounded maps from $X$ to $\mathbf{R}$ with the supremum norm. Let $\sigma$ denote the natural metric on $C(X)$. Let $\lambda_{0}: \mathfrak{L} \rightarrow C(X)$ be a function such that $\lambda_{0}(L) \in i(L)$ for each $L \in \mathfrak{L}$; and define the function $\delta_{0}: \mathfrak{L} \rightarrow(0, \infty)$ by $\delta_{0}(L)=\operatorname{diam} L$ for each $L \in \mathfrak{L}$. Let $\lambda:|n(\mathfrak{L})| \rightarrow C(X)$ and $\delta:|n(\mathfrak{L})| \rightarrow(0, \infty)$ be the linear extensions of $\lambda_{0}$ and $\delta_{0}$, respectively. $\lambda$ and $\delta$ are continuous because $\rho$ is a bounded metric (see Proposition B.2).

Let \|\| denote the natural norm of $l_{1}(\mathfrak{L})$. $|n(\mathfrak{L})|$ lies naturally in the boundary of the unit ball in $l_{1}(\mathfrak{L})$ (see Appendix B). We define the map $\phi:|n(\mathfrak{L})| \rightarrow C(X) \times l_{1}(\mathfrak{L})$ by $\phi(\alpha)=(\lambda(\alpha), \delta(\alpha) \alpha)$ for $\alpha \in|n(\mathfrak{L})| . \phi$ is an embedding; in fact, its inverse is given by the formula $\phi^{-1}(\mu, \beta)=(1 /\|\beta\|) \beta$ for $(\mu, \beta) \in \phi(|n(\mathfrak{L})|)$.

The subset $Z$ of $C(X) \times l_{1}(\mathfrak{L})$ is defined by

$$
Z=(i(A) \times\{0\}) \cup \phi(|n(\mathfrak{L})|) .
$$

The map $g: X \rightarrow Z$ is defined by

$$
g(x)= \begin{cases}(i(x), 0) & \text { for } x \in A \\ \phi \circ \beta(x) & \text { for } x \in X-A\end{cases}
$$

The continuity of $g$ follows from the fact that if $a \in A, \varepsilon>0, x \in X-A$ and $\rho(a, x)<\varepsilon$, then $\sigma(i(a), \lambda \circ \beta(x))<2 \varepsilon$ and $\|(\delta \circ \beta(x)) \beta(x)\|<\varepsilon$. We leave the verification of these inequalities to the reader.

Since $g(A)=Z \cap(C(X) \times\{0\})$, then $g(A)$ is a closed subset of $Z$. Hence $g \mid A: A \rightarrow Z$ is a closed embedding. $Z-g(A)=\phi(|n(\mathfrak{L})|)$ is countable dimensional because $|n(\mathfrak{L})|$ is countable dimension (see Proposition B.5).

5.12. PROPOSITION. If $Y$ is an ANE for the class of all countable dimensional metrizable spaces, then $Y$ has the following property. If $A$ is a countable dimensional closed subset of a metrizable space $X$, then any map $\phi: A \rightarrow Y$ extends to a map $\psi: N \rightarrow Y$ where $N$ is a neighborhood of $A$ in $X$. 
Proof. The preceding lemma provides a map $g: X \rightarrow Z$ into a countable dimensional space $Z$ such that $g \mid A: A \rightarrow Z$ is a closed embedding. Then there is a map $\psi: N \rightarrow Y$ where $N$ is a neighborhood of $g(A)$ in $Z$ and $\psi \mid g(A)=$ $\phi \circ(g \mid A)^{-1}$. Consequently, $g^{-1}(N)$ is a neighborhood of $A$ in $X$, and the map $\psi \circ g \mid g^{-1}(N): g^{-1}(N) \rightarrow Y$ extends $\phi$.

The foregoing results on ANE's for the class of countable dimensional metrizable spaces stimulate the following questions.

5.13. Questions. Consider only metrizable spaces.

(a) Is there an ANE for the class of countable dimensional spaces which is not locally contractible?

(b) Is there is a $U V^{\infty}$ map whose range is not locally contractible?

(c) Does the following property guarantee that a space $Y$ is an ANE for the class of countable dimensional spaces? For every $y \in Y$ and for every neighborhood $U$ of $y$ in $Y$, there is a neighborhood $V$ of $y$ in $Y$ such that each map $f: A \rightarrow V$ from a countable dimensional closed subset $A$ of a space $X$ to $V$ extends to a map from $X$ to $U$.

With the aid of Theorem 5.9, we see that an affirmative answer to Question 5.13(b) entails an affirmative answer to Question 5.13(a). We can frame a more specific form of Question 5.13(b) as follows. Does Taylor's cell-like map which is not a shape equivalence $[\mathbf{T}]$ have a trivial extension to a map whose domain is the Hilbert cube and whose range is not locally contractible? Highly relevant to this question is the following fact from [DW]: if the domain of the Taylor map is embedded as a $\mathbf{Z}$-set in the Hilbert cube, then the range of the associated trivial extension is locally contractible.

The property stated in Question 5.13(c) is possessed by all ANE's for the class of countable dimensional metrizable spaces. A demonstration of this fact is obtained by imitating the proof that (b) implies (c) in Theorem V.2.1, pp. 153-154 of [Hu], with assistance from Proposition 5.12. Thus, Question 5.13(c) asks whether this property is equivalent to being an ANE for the class of countable dimensional spaces.

In our third application, we use the Main Theorem to give a new proof of Haver's theorem in $[\mathbf{H 1}]$ which states that a cell-like map between ANR's is a fine homotopy equivalence. In $[\mathbf{H 1}]$, Haver found it necessary to assume that the domain and range of the cell-like map be separable. Our proof does not require this assumption. This theorem also follows from a theorem of Kozlowski together with Theorem 4.5; for Theorem 9 of $[\mathbf{K 2}]$ states that a cell-like map between ANR's is a hereditary shape equivalence.

5.14. THEOREM. If $f: X \rightarrow Y$ is a cell-like map from an ANR $X$ to an ANR $Y$, then $f$ is a fine homotopy equivalence.

ProOF. Let $\mathfrak{L}$ be an open cover of $Y$. Our goal is to produce a map $g: Y \rightarrow X$ such that $f \circ g \sim 1 \mid Y[\mathfrak{L}]$ and $g \circ f \sim 1 \mid X\left[f^{-1} \mathfrak{L}\right]$.

Let $\mathfrak{M}$ be an open cover of $Y$ such that $\operatorname{star}^{2} \mathfrak{M}$ refines $\mathfrak{L}$ (see A.12). We invoke A.11 and Theorem IV.1.1, p. 111 of $[\mathbf{H u}]$ to obtain an open cover $\mathfrak{N}$ of $Y$ with the following property. If $\phi, \psi: Z \rightarrow Y$ are maps from a topological space $Z$ into $Y$ and $\phi$ is within star $\mathfrak{N}$ of $\psi$, then $\phi \sim \psi[\mathfrak{M}]$.

Now Theorem B.3 provides a polyhedron $P$ with the metric topology and maps $\alpha: Y \rightarrow P$ and $\beta: P \rightarrow Y$ such that $\beta \circ \alpha \sim 1 \mid Y[\mathfrak{N}]$. Since $P$ is countable 
dimensional (Proposition B.5), Main Theorem 3.2 implies that the relation $f^{-1} \circ$ $\beta: P \rightarrow X$ is slice-trivial. A neighborhood $W$ of $f^{-1} \circ \beta$ in $P \times X$ is defined by

$$
W=\bigcup\left\{\beta^{-1} N \times f^{-1} N: N \in \mathfrak{N}\right\} .
$$

Proposition 2.2 (Approximability) provides a map $\gamma: P \rightarrow X$ such that $\gamma \subset W$. It follows that $f \circ \gamma$ is within $\mathfrak{N}$ of $\beta$.

Define the map $g: Y \rightarrow X$ by $g=\gamma \circ \alpha$. Then $f \circ g$ is within $\mathfrak{N}$ of $\beta \circ \alpha$. Since $\beta \circ \alpha$ is within $\mathfrak{N}$ of $1 \mid Y$, then $f \circ g$ is within star $\mathfrak{N}$ of $1 \mid Y$. It follows that there is an $\mathfrak{M}$-homotopy $h: Y \times[0,1] \rightarrow Y$ from $1 \mid Y$ to $f \circ g$.

We use Theorem B.3 again, this time to obtain a polyhedron $Q$ with the metric topology and maps $\zeta: X \rightarrow Q$ and $\eta: Q \rightarrow X$ such that $\left.\eta \circ \zeta \sim 1|X| f^{-1} \mathfrak{M}\right]$. A $U V^{\infty}$ relation $R: Q \times[0,1] \rightarrow X$ is defined by the formula

$$
R=f^{-1} \circ h \circ(f \circ \eta \times 1 \mid[0,1]) .
$$

Main Theorem 3.2 implies that $R$ is slice-trivial, because $Q$ and, therefore, $Q \times[0,1]$ are countable dimensional (see Proposition B.5).

Since $h$ is an $\mathfrak{M}$-homotopy, it follows that $\{R(\{q\} \times[0,1]): q \in Q\}$ refines $f^{-1} \mathfrak{M}$. We shall now produce a neighborhood $U$ of $R$ in $(Q \times[0,1]) \times X$ such that

$$
\{U(\{q\} \times[0,1]): q \in Q\}
$$

refines $f^{-1} \mathfrak{M}$. Let $\pi: Q \times[0,1] \rightarrow Q$ denote projection. Then $R \circ \pi^{-1}: Q \rightarrow X$ is continuous, because $\pi$ is a proper map; and $\left\{R \circ \pi^{-1}(q): q \in Q\right\}$ refines $f^{-1} \mathfrak{M}$. Hence Corollary A.9 provides an open neighborhood $T$ of $R \circ \pi^{-1}$ in $Q \times X$ such that $\{T(q): q \in Q\}$ refines $f^{-1} \mathfrak{M}$. Let $U=T \circ \pi$. Since $R \subset R \circ \pi^{-1} \circ \pi \subset T \circ \pi$ and (using A.1) $T \circ \pi=(\pi \times 1 \mid X)^{-1}(T)$, then $U$ is a neighborhood $R$ in $(Q \times[0,1]) \times X$. Since $U(\{q\} \times[0,1])=T(q)$ for each $q \in Q$, it follows that $\{U(\{q\} \times[0,1]): q \in Q\}$ refines $f^{-1} \mathfrak{M}$.

Define the map $k: Q \times\{0,1\} \rightarrow X$ by the formulas $k(q, 0)=\eta(q)$ and $k(q, 1)=$ $g \circ f \circ \eta(q)$ for $q \in Q$. Since $h_{0}=1 \mid Y$ and $h_{1}=f \circ g$, it follows that $k \subset R$.

Proposition 2.2 (Approximability) provides an open neighborhood $V$ of $R$ in $(Q \times[0,1]) \times X$ with the following property. If $A$ and $B$ are closed subsets of $Q \times[0,1]$ with $A \subset$ int $B$, and if $\phi: B \rightarrow X$ is a map with $\phi \subset V$, then $\phi \mid A$ extends to a map $\phi: Q \times[0,1] \rightarrow X$ with $\Phi \subset U$. We wish to apply this information to extend $k$ to a map $K: Q \times[0,1] \rightarrow X$ such that $K \subset U$. To do this, we must first show that $k$ extends to a map $k_{+}: N \rightarrow X$ where $N$ is a neighborhood of $Q \times\{0,1\}$ in $Q \times[0,1]$ and $k_{+} \subset V$. To this end, let $H=\left[0, \frac{1}{2}\right) \cup\left(\frac{1}{2}, 1\right]$ and define $k_{+}: Q \times H \rightarrow X$ by

$$
k_{+}(q, t)= \begin{cases}k(q, 0) & \text { for }(q, t) \in Q \times\left[0, \frac{1}{2}\right), \\ k(q, 1) & \text { for }(q, t) \in Q \times\left(\frac{1}{2}, 1\right] .\end{cases}
$$

Then $\left(1 \mid Q \times H, k_{+}\right): Q \times H \rightarrow(Q \times H) \times X$ is a map which carries $Q \times\{0,1\}$ onto $k_{+} \mid Q \times\{0,1\}=k$. Since $k \subset R \subset V$, it follows that $N=\left(1 \mid Q \times H, k_{+}\right)^{-1}(V)$ is a neighborhood of $Q \times\{0,1\}$ in $Q \times[0,1]$ and $k_{+} \mid N: N \rightarrow X$ is a map which extends $k$ such that $k_{+} \mid N=\left(1 \mid Q \times H, k_{+}\right)(N) \subset V$. Thus we can legitimately conclude that $k$ extends to a map $K: Q \times[0,1] \rightarrow X$ such that $K \subset U$.

Since $K$ extends $k$ and $K \subset U$, it follow that $K$ is an $f^{-1} \mathfrak{M}$-homotopy from $\eta$ to $g \circ f \circ \eta$. Thus

$$
K \circ(\varsigma \times 1 \mid[0,1]): X \times[0,1] \rightarrow X
$$


is an $f^{-1} \mathfrak{M}$-homotopy from $\eta \circ \varsigma$ to $g \circ f \circ \eta \circ \zeta$.

Since $f \circ g$ is within $\mathfrak{M}$ of $1 \mid Y$, then $g \circ f$ is within $f^{-1} \mathfrak{M}$ of $1 \mid X$. It follows that for each $M \in \mathfrak{M}, g \circ f\left(f^{-1} M\right) \subset f^{-1}(\operatorname{star}(M, \mathfrak{M}))$. As a result, the composition of $g \circ f$ with the homotopy $\eta \circ \zeta \sim 1 \mid X\left[f^{-1} \mathfrak{M}\right]$ yields a homotopy $g \circ f \circ \eta \circ \zeta \sim g \circ f\left[f^{-1} \operatorname{star} \mathfrak{M}\right]$.

The homotopies $1 \mid X \sim \eta \circ \varsigma\left[f^{-1} \mathfrak{M}\right], \eta \circ \varsigma \sim g \circ f \circ \eta \circ \varsigma\left[f^{-1} \mathfrak{M}\right]$ and $g \circ f \circ \eta \circ \varsigma \sim$ $g \circ f\left[f^{-1}\right.$ star, $\left.\mathfrak{M}\right]$ string together to yield a homotopy $1 \mid X \sim g \circ f\left[f^{-1} \operatorname{star}^{2} \mathfrak{M}\right]$. Hence $1 \mid X \sim g \circ f\left[f^{-1} \mathfrak{L}\right]$. Since $1 \mid Y \sim f \circ g[\mathfrak{L}]$ as well, we conclude that $f$ is a fine homotopy equivalence.

Appendix A. Continuous relations. Most of the ideas in this section originally appeared in $[\mathbf{C 1}]$. Enlargement Lemma A.8 was first stated in $[\mathbf{A}]$.

If $X$ and $Y$ are sets and $R \subset X \times Y$, then we call $R$ a relation from $X$ to $Y$ and we write $R: X \rightarrow Y$. The identity relation on a set $X$, denoted $1 \mid X: X \rightarrow X$, is defined by

$$
1 \mid X=\{(x, x) \in X \times X: x \in X\} \text {. }
$$

If $R: X \rightarrow Y$ and $S: Y \rightarrow Z$ are relations, then the composition of $R$ and $S$, denoted $S \circ R: X \rightarrow Z$, is defined by

$$
S \circ R=\{(x, z) \in X \times Z:(x, y) \in R \text { and }(y, z) \in S \text { for some } y \in Y\} .
$$

The inverse of a relation $R: X \rightarrow Y$, denoted $R^{-1}: Y \rightarrow X$, is defined by

$$
R^{-1}=\{(y, x) \in Y \times X:(x, y) \in R\} .
$$

If $R: W \rightarrow Y$ and $S: X \rightarrow Z$ are relations, then the cartesian product of $R$ and $S$, denoted $R \times S:(W \times X) \rightarrow(Y \times Z)$ is defined by

$$
R \times S=\{((w, x),(y, z)) \in(W \times X) \times(Y \times Z):(w, y) \in R \text { and }(x, z) \in S\} .
$$

If $R: X \rightarrow Y$ and $S: X \rightarrow Z$ are relations, then the ordered pair relation $(R, S): X \rightarrow$ $(Y \times Z)$ is defined by

$$
(R, S)=\{(x,(y, z)) \in X \times(Y \times Z):(x, y) \in R \text { and }(x, z) \in S\} ;
$$

observe that $(R, S)=(R \times S) \circ \Delta$ where $\Delta: X \rightarrow X \times X$ is the diagonal relation defined by $\Delta(x)=(x, x)$ for each $x \in X$.

A.1. Proposition. If $Q: W \rightarrow X, R: X \rightarrow Y$ and $S: Y \rightarrow Z$ are relations, then the following identities hold:

(1) $R \circ(1 \mid X)=R=(1 \mid Y) \circ R$.

(2) $(S \circ R)^{-1}=R^{-1} \circ S^{-1}$.

(3) $(S \circ R) \circ Q=S \circ(R \circ Q)=\left(Q^{-1} \times S\right)(R)$.

The third identity, though perhaps unfamiliar, is quite useful.

Let $R: X \rightarrow Y$ be a relation. For each $x \in X$, let $R(x)=\{y \in Y:(x, y) \in$ $R\} ; R(x)$ is called a point image of $R$. For each $A \subset X$, let $R(A)=\bigcup\{R(x): x \in A\}$. It follows that for each $y \in Y, R^{-1}(y)=\{x \in X:(x, y) \in R\}$ and that for each $B \subset$ $Y, R^{-1}(B)=\bigcup\left\{R^{-1}(y): y \in B\right\}$. The domain of the relation $R: X \rightarrow Y$, denoted $\operatorname{dom} R$, is defined by $\operatorname{dom} R=\{x \in X: R(x) \neq \emptyset\}$; thus $\operatorname{dom} R=R^{-1}(Y)$. For each $x \in X$, the slice of $R$ over $x$, denoted $R \mid x$, is defined by $R \mid x=R \cap(\{x\} \times Y)$. For each $A \subset X$, the restriction of $R$ to $A$, denoted $R \mid A: A \rightarrow Y$, is defined by $R \mid A=R \cap(A \times Y)$. 
Let $R: X \rightarrow Y$ be a relation from a topological space $X$ to a topological space $Y$. $R: X \rightarrow Y$ is continuous if for each $x \in X$ and each neighborhood $M$ of $R(x)$ in $Y$, there is a neighborhood $L$ of $x$ in $X$ such that $R(L) \subset M . R: X \rightarrow Y$ has compact point images if for each $x \in X$, the point image $R(x)$ is a compact (possibly empty) subset of $Y$.

The following five facts have simple proofs which are left to the reader.

A.2. Proposition. A relation $R: X \rightarrow Y$ from a topological space $X$ to $a$ topological space $Y$ is continuous if and only if $R^{-1}(B)$ is a closed subset of $X$ whenever $B$ is a closed subset of $Y$.

A.3. Proposition. Suppose $X$ and $Y$ are topological spaces and $R: X \rightarrow Y$ is a continuous relation with compact point images. If $A$ is a compact subset of $X$, then $R(A)$ is a compact subset of $Y$.

A.4. Corollary. Let $X, Y$, and $Z$ be topological spaces. If $R: X \rightarrow Y$ and $S: Y \rightarrow Z$ are continuous relations (with compact point images), then so is $S \circ R: X \rightarrow Z$.

A.5. Proposition. Suppose $W, X, Y$ and $Z$ are topological spaces. If $R: W \rightarrow$ $Y$ and $S: X \rightarrow Z$ are continuous relations with compact point images, then so is $R \times S:(W \times X) \rightarrow(Y \times Z)$.

A.6. Corollary. Suppose $X, Y$ and $Z$ are topological spaces. If $R: X \rightarrow Y$ and $S: X \rightarrow Z$ are continuous relations with compact point images, then so is $(R, S): X \rightarrow(Y \times Z)$.

A.7. PROPOSITION. If $R: X \rightarrow Y$ is a continuous relation with compact point images from a topological space $X$ to a Hausdorff space $Y$, then $R$ is a closed subset of $X \times Y$.

The next result is a particularly useful aid in the study of continuous relations.

A.8. Enlargement Lemma. Suppose $X$ is a paracompact regular space, $n$ is a positive integer, and for each $i, 1 \leq i \leq n, R_{i}: X \rightarrow Y_{i}$ is a continuous relation from $X$ to a topological space $Y_{i}$. Further suppose that for each $i, 1 \leq i \leq n$, and each $x \in X, N_{i, x}$ is an open neighborhood of $R_{i}(x)$ in $Y_{i}$. Let $\left\{J_{x}: x \in X\right\}$ be an open cover of $X$ such that $x \in J_{x}$ for each $x \in X$. Then there is an open cover $\left\{L_{x}: x \in X\right\}$ of $X$ and there is for each $i, 1 \leq i \leq n$, an open neighborhood $U_{i}$ of $R_{i}$ in $X \times Y_{i}$ such that $L_{x} \subset J_{x}$ and $U_{i}\left(L_{x}\right) \subset N_{i, x}$ for each $x \in X$. ( $U_{i}$ is called an enlargement of $R_{i}$, for $1 \leq i \leq n$.)

PROOF. Using the hypotheses that each $R_{i}$ is continuous and that $X$ is paracompact and regular, we obtain a locally finite open cover $\left\{L_{x}: x \in X\right\}$. of $X$ such that for each $x \in X, L_{x} \subset J_{x}$ and $R_{i}\left(\operatorname{cl} L_{x}\right) \subset N_{i, x}$ for $1 \leq i \leq n$.

Fix $i, 1 \leq i \leq n$. Define $U_{i}: X \rightarrow Y_{i}$ by specifying that for each $x \in X$,

$$
U_{i}(x)=\bigcap\left\{N_{i, w}: w \in X \text { and } x \in \operatorname{cl} L_{w}\right\} .
$$

Let $x \in X$. To see that $U_{i}\left(L_{x}\right) \subset N_{i, x}$, observe that if $z \in L_{x}$, then $U_{i}(z) \subset N_{i, x}$. To see that $R_{i}(x) \subset U_{i}(x)$, observe that if $w \in X$ and $x \in \operatorname{cl} L_{w}$, then $R_{i}(x) \subset$ $R_{i}\left(\operatorname{cl} L_{w}\right) \subset N_{i, w}$. It follows that $R_{i} \subset U_{i}$. 
It remains to show that $U_{i}$ is an open subset of $X \times Y_{i}$. Let $x \in X$. The local finiteness of $\left\{L_{w}: w \in X\right\}$ implies that $U_{i}(x)$ is an open subset of $Y_{i}$ and that $M_{x}=$ $X-\bigcup\left\{\operatorname{cl} L_{w}: w \in X\right.$ and $\left.x \notin \operatorname{cl} L_{w}\right\}$ is an open neighborhood of $x$ in $X$. We assert that $M_{x} \times U_{i}(x) \subset U_{i}$. The definition of $M_{x}$ implies that for any $v \in M_{x}$ : if $w \in X$ and $v \in \operatorname{cl} L_{w}$, then $x \in \operatorname{cl} L_{w}$. It follows that $U_{i}(x) \subset U_{i}(v)$ for each $v \in M_{x}$. This establishes our assertion. The assertion implies that $U_{i}=\bigcup\left\{M_{x} \times U_{i}(x): x \in X\right\}$. We conclude that $U_{i}$ is an open subset of $X \times Y_{i}$.

Specializing the Enlargement Lemma to the case of one relation, we have

A.9. COROLlaRY. Suppose $X$ is a paracompact regular space and $R: X \rightarrow Y$ is a continuous relation from $X$ to a topological space $Y$. For every collection $\mathfrak{N}$ of open subsets of $Y$ which is refined by $\{R(x): x \in X\}$, there is an open neighborhood $U$ of $R$ in $X \times Y$ such that $\{U(x): x \in X\}$ refines $\mathfrak{N}$.

The next lemma is an important and useful consequence of the Enlargement Lemma.

A.10. COMPOSITION LEMMA. Let $X$ be a paracompact regular space, let $Y$ and $Z$ be topological spaces, and let $R: X \rightarrow Y$ and $S: X \rightarrow Z$ be continuous relations with compact point images. For every open neighborhood $U$ of $S \circ R^{-1}$ in $Y \times Z$, there are open neighborhoods $V$ of $R$ in $X \times Y$ and $W$ of $S$ in $X \times Z$ such that $W \circ V^{-1} \subset U$.

PROOF. For each $x \in X$, since $R(x)$ and $S(x)$ are compact and $R(x) \times S(x) \subset$ $S \circ R^{-1} \subset U$, then there are open neighborhoods $M_{x}$ of $R(x)$ in $Y$ and $N_{x}$ of $S(x)$ in $Z$ such that $M_{x} \times N_{x} \subset U$. We invoke Enlargement Lemma A.8 to obtain an open cover $\left\{L_{x}: x \in X\right\}$ of $X$ and open neighborhoods $V$ of $R$ in $X \times Y$ and $W$ of $S$ in $X \times Z$ such that $V\left(L_{x}\right) \subset M_{x}$ and $W\left(L_{x}\right) \subset N_{x}$ for each $x \in X$. For each $x \in X$, there is a $w \in X$ such that $x \in L_{w}$; then

$$
V(x) \times W(x) \subset V\left(L_{w}\right) \times W\left(L_{w}\right) \subset M_{w} \times N_{w} \subset U .
$$

Since $W \circ V^{-1}=\bigcup\{V(x) \times W(x): x \in X\}$, it follows that $W \circ V^{-1} \subset U$.

We close this appendix by demonstrating how these techniques provide an entertaining new proof of an old theorem. Let $\mathfrak{L}$ be a collection of subsets of a set $X$. For each subset $A$ of $X$, the subset $\operatorname{star}(A, \mathfrak{L})$ of $X$ is defined by $\operatorname{star}(A, \mathfrak{L})=\bigcup\{L \in \mathfrak{L}: A \cap L \neq \emptyset\}$. The collection star $\mathfrak{L}$ of subsets of $X$ is defined by $\operatorname{star} \mathfrak{L}=\{\operatorname{star}(L, \mathfrak{L}): L \in \mathfrak{L}\}$. We say that $\mathfrak{L}$ star-refines a collection $\mathfrak{M}$ of subsets of $X$ if star $\mathfrak{L}$ refines $\mathfrak{M}$.

A.11. COROllaRY. Every open cover of a paracompact regular space $X$ is star-refined by another open cover of $X$.

ProOF. Let $\mathfrak{L}$ be an open cover of $X$. A.9 provides an open neighborhood $U$ of $1 \mid X$ in $X \times X$ such that $\{U(x): x \in X\}$ refines $\mathfrak{L}$. A.10 provides an open neighborhood $V$ of $1 \mid X$ in $X \times X$ such that $V \circ V^{-1} \circ V \subset U$. Then $\mathfrak{M}=$ $\{V(x): x \in X\}$ is an open cover of $X$ which star-refines $\mathfrak{L}$.

Let $\mathfrak{L}$ be a collection of subsets of a set $X$. For each subset $A$ of $X$ and each integer $n \geq 0$, we define the subset $\operatorname{star}^{n}(A, \mathfrak{L})$ of $X$ inductively by setting $\operatorname{star}^{0}(A, \mathfrak{L})=A$ and setting $\operatorname{star}^{n+1}(A, \mathfrak{L})=\operatorname{star}\left(\operatorname{star}^{n}(A, \mathfrak{L}), \mathfrak{L}\right)$ for each $n \geq 0$. For each $n \geq 0$, we define the collection $\operatorname{star}^{n} \mathfrak{L}$ of subsets of $X$ by $\operatorname{star}^{n} \mathfrak{L}=\left\{\operatorname{star}^{n}(L, \mathfrak{L}): L \in \mathfrak{L}\right\}$. A simple induction proof based on Corollary 
A.11 shows that

A.12. COROLLARY. Let $\mathfrak{L}$ be an open cover of a paracompact regular space $X$. For every integer $n \geq 0$, there is an open cover $\mathfrak{M}$ of $X$ such that $\operatorname{star}^{n} \mathfrak{M}$ refines $\mathfrak{L}$.

Appendix B. Polyhedra with the metric topology. The polyhedron underlying the nerve of a cover plays a crucial role in the theory of ANR's. Such a polyhedron is usually assigned one of two competing topologies: either the Whitehead topology which is not in general metrizable, or the metric topology which (as the name suggests) is always metrizable. Since the developments in this paper are primarily restricted to metrizable spaces, we prefer to deal with polyhedra endowed with the metric topology. Unfortunately in the standard expositions of the theory of ANR's (such as $[\mathbf{H u}]$ ), the properties of polyhedra are developed more extensively for the Whitehead topology than for the metric topology. This appendix aims to repair this omission by indicating why the properties of polyhedra which are important for the study of ANR's and which are known to hold under the Whitehead topology, also hold under the metric topology.

We first recall the basic definitions.

Let $V$ be a set. An abstract simplicial complex with vertex set $V$ is a collection $K$ of nonempty finite subsets of $V$ such that:

(1) $\{v\} \in K$ for each $v \in V$, and

(2) if $\sigma \in K$ and $\emptyset \neq \tau \subset \sigma$, then $\tau \in K$.

Nerves provide an important class of examples of abstract simplicial complexes. Let $\mathfrak{L}$ be a collection of nonempty subsets of a set $X$. The nerve of $\mathfrak{L}$, denoted $n(\mathfrak{L})$, is the abstract simplicial complex with vertex set $\mathfrak{L}$ consisting of all nonempty finite subsets of $\mathfrak{L}$ which have nonempty intersection.

Let $K$ be an abstract simplicial complex with vertex set $V$. An element of $V$ is called a vertex of $K$, and an element of $K$ is called a simplex of $K$. A subset of $K$ which is itself an abstract simplicial complex is called a subcomplex of $K$; the vertex set of a subcomplex of $K$ is a subset of $V$. If a simplex $\sigma$ of $K$ contains exactly $n+1$ elements of $V$, then $\sigma$ is called an $n$-simplex and we write $\operatorname{dim} \sigma=n$. For each $n \geq 0$, the $n$-skeleton of $K$, denoted $K^{n}$, is the subcomplex of $K$ with vertex set $V$ defined by $K^{n}=\{\sigma \in K: \operatorname{dim} \sigma \leq n\}$. Thus $K=\bigcup_{n=1}^{\infty} K^{n}$.

Let $K$ be an abstract simplicial complex with vertex set $V$. The polyhedron underlying $K$, denoted $|K|$, is the set of all functions $\alpha: V \rightarrow[0,1]$ such that:

(1) $\alpha^{-1}(0,1] \in K$ and

(2) $\sum\{\alpha(v): v \in V\}=1$.

If $\sigma$ is a simplex of $K$, then define

$$
\begin{gathered}
|\sigma|=\left\{\alpha \in|K|: \alpha^{-1}(0,1] \subset \sigma\right\} \\
\partial|\sigma|=\left\{\alpha \in|K|: \alpha^{-1}(0,1] \subset \sigma \text { and } \alpha^{-1}(0,1] \neq \sigma\right\}
\end{gathered}
$$

and

$$
\operatorname{int}|\sigma|=|\sigma|-\partial|\sigma|=\left\{\alpha \in|K|: \alpha^{-1}(0,1]=\sigma\right\} .
$$

If $L$ is a subcomplex of $K$, then define

$$
|L|=\left\{\alpha \in|K|: \alpha^{-1}(0,1] \in L\right\} .
$$

Thus $|K|=\bigcup\{$ int $|\sigma|: \sigma \in K\}=\bigcup_{n=1}^{\infty}\left|K^{n}\right|$. 
A polyhedron is any set of the form $|K|$ where $K$ is some abstract simplicial complex.

Let $K$ be an abstract simplicial complex with vertex set $V$. The metric topology on $|K|$ is the topology determined by the following requirement. A sequence $\left\{\alpha_{n}\right\}$ of points of $|K|$ converges to a point $\beta$ of $|K|$ if and only if $\left\{\alpha_{n}(v)\right\}$ converges to $\beta(v)$ in $[0,1]$ for each $v \in V$. This requirement is equivalent to saying that $|K|$ be regarded as a subspace of the space $[0,1]^{V}$ of all functions from $V$ to $[0,1]$ endowed with the product topology. Many different metrics induce the metric topology on $|K|$. For instance, the following three formulas define metrics $\rho_{1}, \rho_{2}$ and $\rho_{\infty}$ on $|K|$ which induce the metric topology:

$$
\begin{aligned}
\rho_{1}(\alpha, \beta) & =\sum\{|\alpha(v)-\beta(v)|: v \in V\}, \\
\rho_{2}(\alpha, \beta) & =\left(\sum\left\{(\alpha(v)-\beta(v))^{2}: v \in V\right\}\right)^{1 / 2}, \\
\rho_{\infty}(\alpha, \beta) & =\sup \{|\alpha(v)-\beta(v)|: v \in V\}
\end{aligned}
$$

for $\alpha, \beta \in|K|$. For our purposes, it is most convenient to take the metric $\rho_{1}$ as the standard metric on $|K|$. Let $l_{1}(V)$ denote the Banach space of all functions $\alpha: V \rightarrow \mathbf{R}$ satisfying $\sum\{|\alpha(v)|: v \in V\}<\infty$, with the norm \|\| defined by $\|\alpha\|=$ $\sum\{|\alpha(v)|: v \in V\}$ for $\alpha \in l_{1}(V)$. Then $|K|$ includes naturally into $l_{1}(V) .|K|$ is a subset of the boundary of the unit ball in $l_{1}(V)$; i.e., $\|\alpha\|=1$ for every $\alpha \in|K|$. $\rho_{1}$ is the restriction to $|K|$ of the natural norm-induced metric on $l_{1}(V)$.

Let $K$ be an abstract simplicial complex with vertex set $V$. The Whitehead topology on $|K|$ is the topology determined by the following requirement. A subset $O$ of $|K|$ is open if and only if for every simplex $\sigma$ of $K, O \cap|\sigma|$ is a (relatively) open subset of $|\sigma|$ where $|\sigma|$ is regarded as a subspace of $|K|$ endowed with the metric topology. In general, the Whitehead topology is finer than the metric topology. The two topologies coincide (and the Whitehead topology is metrizable) if and only if $K$ is locally finite. ( $K$ is locally finite if each vertex of $K$ is contained in only a finite number of simplices of $K$.)

Let $\mathfrak{L}$ be an open cover of a topological space $X$. Let $|n(\mathfrak{L})|$, the polyhedron underlying the nerve of $\mathfrak{L}$, be endowed with either the Whitehead or the metric topology. A map $\beta: X \rightarrow|n(\mathfrak{L})|$ is called a barycentric map if for each $L \in \mathfrak{L},\{x \in$ $X: \beta(x)(L) \neq 0\} \subset L$. If $\mathfrak{L}$ is an open cover of a metrizable space $X$, then Theorem VIII.5.4, p. 172 of $[\mathbf{D}]$ provides a barycentric map $\beta: X \rightarrow|n(\mathfrak{L})|$ with respect to the Whitehead topology on $|n(\mathfrak{L})|$. Since the Whitehead topology is finer than the metric topology, then $\beta$ is also a barycentric map with respect to the metric topology on $\mid(n(\mathfrak{L}) \mid$. Thus we have

B.1. PROPOSITION. If $\mathfrak{L}$ is an open cover of a metrizable space $X$, and $|n(\mathfrak{L})|$ is endowed with the metric topology, then there is a barycentric map from $X$ to $|n(\mathfrak{L})|$.

Let $K$ be an abstract simplicial complex with vertex set $V$. The Whitehead topology on $|K|$ has the virtue (not shared by the metric topology) that there is a very simple criterion for determining whether a function with domain $|K|$ is continuous. Such a function is continuous if its restriction to $|\sigma|$ is continuous for each $\sigma \in K$. We now develop a continuity criterion for certain maps with domain $|K|$ with respect to the metric topology. 
Let $K$ be an abstract simplicial complex with vertex set $V$. Let $Y$ be a normed linear space. If $\lambda_{0}: V \rightarrow Y$ is a function, then the linear extension of $\lambda_{0}$ is the function $\lambda:|K| \rightarrow Y$ defined by $\lambda(\alpha)=\sum\left\{\alpha(v) \lambda_{0}(v): v \in V\right\}$ for each $\alpha \in|K|$. With the Whitehead topology on $|K|$, the linear extension of any function from $V$ to $Y$ is continuous. This is not true for the metric topology. However, there is a simple criterion which determines whether the linear extension of a function from $V$ to $Y$ is continuous with respect to the metric topology on $|K|$.

B.2. PROPOSITION. Let $K$ be an abstract simplicial complex with vertex set $V$, and let $Y$ be a normed linear space. The linear extension of a function $\lambda_{0}: V \rightarrow Y$ is continuous with respect to the metric topology on $|K|$ if and only if for each $v \in V,\left\{\lambda_{0}\left(v^{\prime}\right): v^{\prime} \in V\right.$ and $\left.\left\{v, v^{\prime}\right\} \in K\right\}$ is a bounded subset of $Y$.

The proof is left to the reader.

We now come to one of the fundamental results relating polyhedra and ANR's.

B.3. THEOREM. Let $X$ be an ANR. For every open cover $\mathfrak{L}$ of $X$, there is a polyhedron $P$ endowed with the metric topology and maps $\phi: X \rightarrow P$ and $\psi: P \rightarrow X$ such that $\psi \circ \phi \sim 1 \mid X[\mathfrak{L}]$. (This notation is explained at the beginning of $\S 4$.)

This is proved for a polyhedron with the Whitehead topology in Theorem IV.6.1, p. 138 of $[\mathbf{H u}]$. It does not follow automatically for polyhedra with the metric topology because $\psi: P \rightarrow X$ is not necessarily continuous. We shall present a proof for the case of the metric topology.

PROOF. Let $\mathfrak{L}$ be an open cover of $X$.

Let $\rho$ be a bounded metric on $X$. Theorem XIII.5.2, p. 286 of $[\mathbf{D}]$ provides an isometric embedding $i: X \rightarrow C(X)$, where $C(X)$ denotes the Banach space of all bounded maps from $X$ to $\mathbf{R}$ with the supremum norm, such that $i(X)$ is a closed subset of its convex hull $Z$ in $C(X)$. Since $X$ is an ANR, there is a (relatively) open neighborhood $O$ of $i(X)$ in $Z$ and a map $r: O \rightarrow X$ such that $r \circ i=1 \mid X$.

Let $\mathfrak{C}$ be a collection of convex (relatively) open subsets of $O$ which covers $i(X)$ and refines $r^{-1} \mathfrak{L}$. Let $\mathfrak{M}$ be an open cover of $X$ which star-refines $i^{-1} \mathfrak{C}$ (use A.11).

We endow $|n(\mathfrak{M})|$, the polyhedron underlying the nerve of $\mathfrak{M}$, with the metric topology. Proposition B.1 provides a barycentric map $\phi: X \rightarrow|n(\mathfrak{M})|$.

Let $\lambda_{0}: \mathfrak{M} \rightarrow i(X)$ be a function such that $\lambda_{0}(M) \in i(M)$ for each $M \in \mathfrak{M}$. Let $\lambda:|n(\mathfrak{M})| \rightarrow C(X)$ be the linear extension of $\lambda_{0}$. Since the metric $\rho$ on $X$ is bounded and $i$ is an isometry, then Proposition B.2 implies that $\lambda$ is continuous.

If $\sigma \in n(\mathfrak{M})$, then $\lambda_{0}(\sigma) \subset i(\bigcup \sigma) \subset C$ for some $C \in \mathfrak{C}$; since $C$ is convex, it follows that $\lambda(|\sigma|) \subset C$. Consequently, $\lambda(|n(\mathfrak{M})|) \subset \bigcup \mathfrak{C} \subset 0$. Hence we can define the $\operatorname{map} \psi:|n(\mathfrak{M})| \rightarrow X$ by $\psi=r \circ \lambda$.

Let $h: X \times[0,1] \rightarrow C(X)$ be the straight-line homotopy from $i$ to $\lambda \circ \phi$; i.e., $h(x, t)=(1-t) i(x)+t(\lambda \circ \phi(x))$ for $(x, t) \in X \times[0,1]$.

Let $x \in X$. Let $\sigma=\{M \in \mathfrak{M}: \phi(x)(M) \neq 0\}$. Then $\sigma \in n(\mathfrak{M}), x \in \bigcap \sigma$ and $\phi(x) \in|\sigma|$. There is a $C \in \mathfrak{C}$ such that $i(\bigcup \sigma) \subset C$. Then $i(x) \in C$ and $\lambda_{0}(\sigma) \subset C$. Since $C$ is convex, $\lambda(|\sigma|) \subset C$. So $\lambda \circ \phi(x) \in C$. Therefore $h(\{x\} \times[0,1]) \subset C$.

We have proved that $h$ is a $\mathfrak{C}$-homotopy from $i$ to $\lambda \circ \phi$. Since $\mathfrak{C}$ refines $r^{-1} \mathfrak{L}$, it follows that $r \circ h: X \times[0,1] \rightarrow X$ is an $\mathfrak{L}$-homotopy from $r \circ i=1 \mid X$ to $r \circ \lambda \circ \phi=\psi \circ \phi$.

For our purposes, it is essential to know that a polyhedron with the metric topology is countable dimensional. This fact follows from the next lemma. 
B.4. LEMMA. Let $K$ be an abstract simplicial complex. Endow $|K|$ with the metric topology. Then $\operatorname{dim}\left|K^{n}\right| \leq n$ for each $n \geq 0$.

ProOF. We proceed by induction on $n$. Since each point of $\left|K^{0}\right|$ is isolated, $\operatorname{dim}\left|K^{0}\right|=0$.

Let $n \geq 1$ and assume $\operatorname{dim}\left|K^{n-1}\right| \leq n-1$. $\left|K^{n}\right|$ is the union of the closed set $\left|K^{n-1}\right|$ and the relatively open set $\left|K^{n}\right|-\left|K^{n-1}\right|$. Since $\operatorname{dim}\left|K^{n-1}\right| \leq n-1$, and since $\left|K^{n}\right|-\left|K^{n-1}\right|$ is the union of a countable collection of closed subsets of $\left|K^{n}\right|$, then Theorem II.2.C, p. 16 of $[\mathbf{N}]$ implies that it suffices to prove that $\operatorname{dim}\left(\left|K^{n}\right|-\left|K^{n-1}\right|\right) \leq n$. Now $\{\operatorname{int}|\sigma|: \sigma \in K$ and $\operatorname{dim} \sigma=n\}$ is a disjoint collection of (relatively) open subsets of $\left|K^{n}\right|-\left|K^{n-1}\right|$ whose union is $\left|K^{n}\right|-\left|K^{n-1}\right|$. Thus \{int $|\sigma|: \sigma \in K$ and $\operatorname{dim} \sigma=n\}$ is a locally finite collection of (relatively) closed subsets of $\left|K^{n}\right|-\left|K^{n-1}\right|$. Hence, by Theorem II.2.D, p. 16 of [N], it suffices to show $\operatorname{dim}(\operatorname{int}|\sigma|) \leq n$ for each $\sigma \in K$ with $\operatorname{dim} \sigma=n$.

Let $\sigma \in K$ with $\operatorname{dim} \sigma=n$. Say $\sigma=\left\{v_{0}, v_{1}, \ldots, v_{n}\right\}$. We shall prove $\operatorname{dim}(\operatorname{int}|\sigma|)$ $\leq n$ by exhibiting a homeomorphism $h_{\sigma}$ : int $|\sigma| \rightarrow W$ where $W$ is the set of all points $\left(x_{1}, \ldots, x_{n}\right)$ of $\mathbf{R}^{n}$ such that $x_{i}>0$ for $1 \leq i \leq n$ and $\sum_{i=1}^{n} x_{i}<1$. Then $\operatorname{dim}(\operatorname{int}|\sigma|) \leq n$ will follow from [N, Theorem IV.4, p. 97]. Define $h_{\sigma}$ : int $|\sigma| \rightarrow W$ by $h_{\sigma}(\alpha)=\left(\alpha\left(v_{1}\right), \ldots, \alpha\left(v_{n}\right)\right)$ for $\alpha \in \operatorname{int}|\sigma|$. To see that $h_{\sigma}$ is a homeomorphism, observe that its inverse $h_{\sigma}^{-1}: W \rightarrow$ int $|\sigma|$ is given by the formula

$$
h_{\sigma}^{-1}\left(x_{1}, \ldots, x_{n}\right)(v)= \begin{cases}x_{i} & \text { if } v=v_{i} \text { for } 1 \leq i \leq n \\ 1-\sum_{i=1}^{n} x_{i} & \text { if } v=v_{0} \\ 0 & \text { if } v \in V-\sigma\end{cases}
$$

for $\left(x_{1}, \ldots, x_{n}\right) \in W$.

Since $|K|=\bigcup_{n=0}^{\infty}\left|K^{n}\right|$ for each abstract simplicial complex $K$, then the preceding lemma implies

B.5. Proposition. Every polyhedron with the metric topology is countable dimensional.

Finally we mention an important fact established in Theorem III.11.3, p. 106 of $[\mathbf{H u}]$.

B.6. PROPOSITION. Every polyhedron with the metric topology is an ANR.

Appendix C. Property C. A topological space $X$ has property $\mathrm{C}$ if for each sequence $\left\{\mathfrak{L}_{i}: i \geq 1\right\}$ of open covers of $X$, there is an open cover of $X$ of the form $\bigcup_{i=1}^{\infty} \mathfrak{M}_{i}$ such that for each $i \geq 1, \mathfrak{M}_{i}$ is a pairwise disjoint collection which refines $\mathfrak{L}_{i}$. Property $\mathrm{C}$ was first formulated by W. E. Haver in [H2]. Lemma 3.3 implies that all countable dimensional metrizable spaces have property $\mathrm{C}$. The question of the existence of a noncountable dimensional metrizable space with property $\mathrm{C}$ was recently settled by $R$. Pol: $[\mathbf{P}]$ exhibits an ingenious construction of a compact metrizable space which has property $\mathrm{C}$ but is not countable dimensional. Consequences of property $C$ have been studied further in $[\mathbf{H 3}, \mathbf{H 4}, \mathbf{A G}$ and $\mathbf{G}]$.

This appendix consists of strengthened versions of Main Theorem 3.2 and a number of its corollaries, obtained by replacing the hypothesis of countable dimensionality by property $\mathrm{C}$. Each result in this appendix is numbered by prefixing a "C" to the number of the corresponding unstrengthened result which occurs earlier in the paper. 
We begin by generalizing Main Theorem 3.2. The only change needed in the proof of the Main Theorem is the following modification of Lemma 3.3.

C.3.3. Lemma. Let $X$ be a metrizable space, and let $Z$ be a subspace of $X$ with property $\mathrm{C}$. If for each $i \geq 1, \mathfrak{L}_{i}$ is a collection of open subsets of $X$ which covers $Z$, then there is a collection of open subsets of $X$ which covers $Z$ and which has the form $\bigcup_{i=1}^{\infty} \mathfrak{M}_{i}$ where for each $i \geq 1, \mathfrak{M}_{i}$ is a pairwise disjoint collection which refines $\mathfrak{L}_{i}$.

PROOF. Since $Z$ has property C, there is a collection of relatively open subsets of $Z$ which covers $Z$ and which has the form $\bigcup_{i=1}^{\infty} \mathfrak{N}_{i}$ where for each $i \geq 1, \mathfrak{N}_{i}$ is a pairwise disjoint collection which refines $\mathfrak{L}_{i}$. Let $i \geq 1$. For each $N \in \mathfrak{N}_{i}$, select $L(N) \in \mathfrak{L}_{i}$ and define $M(N)$ as in the proof of Lemma 3.3. Then define $\mathfrak{M}_{i}$ as in that proof. Therefore, $\mathfrak{M}_{i}$ is a pairwise disjoint collection of open subsets of $X$ which refines $\mathfrak{L}_{i}$ and is refined by $\mathfrak{N}_{i}$. Consequently, $\bigcup_{i=1}^{\infty} \mathfrak{M}_{i}$ covers $Z$.

By allowing Lemma C.3.3 to take over the role of Lemma 3.3, the proof of Main Theorem 3.2 is transformed into a proof of the following result.

C.3.2. Generalized MAIN TheOREM. Let $R: X \rightarrow Y$ be a $U V^{\infty}$ relation from a metrizable space $X$ to a metrizable space $Y$. If there is a sequence $\left\{K_{i}\right\}$ of closed subsets of dom $R$ such that

(1) $\operatorname{dom} R-\bigcup_{i=1}^{\infty} K_{i}$ has property $\mathrm{C}$, and

(2) $R \mid K_{i}$ is slice-trivial in $X \times Y$ for each $i \geq 1$, then $R$ is slice-trivial.

If, in the proofs in $\S 5$, property $\mathrm{C}$ is substituted for countable dimensionality, and Theorem C.3.2 is used in place of Theorem 3.2, then the following results are obtained.

C.5.1. THEOREM. A cell-like map $f: X \rightarrow Y$ from a metrizable space $X$ to a metrizable space $Y$ is a hereditary shape equivalence if there is a sequence $\left\{K_{n}\right\}$ of closed subsets of $Y$ such that

(1) $Y-\bigcup_{n=1}^{\infty} K_{n}$ has property $\mathrm{C}$, and

(2) $f \mid f^{-1}\left(K_{n}\right): f^{-1}\left(K_{n}\right) \rightarrow K_{n}$ is a hereditary shape equivalence for each $n \geq 1$.

C.5.2. Corollary. A cell-like map between metrizable spaces is a hereditary shape equivalence if its singular set is contained in $a G_{\delta}$ subset of its range which has property $\mathrm{C}$.

C.5.3. COROllary. A cell-like map between metrizable spaces is a hereditary shape equivalence if its range has property $\mathrm{C}$.

C.5.9. THEOREM. Let $f: X \rightarrow Y$ be a $U V^{\infty}$ map from a metrizable space $X$ to a metrizable space $Y$. Then $Y$ is an ANE for the class of all metrizable spaces with property $\mathrm{C}$. Furthermore, if $Y$ has property $\mathrm{C}$, then $Y$ is an $A N R$.

C.5.10. COROLlaRY. Every locally contractible metrizable space is an ANE for the class of all metrizable spaces with property C. Furthermore, every locally contractible metrizable space which has property $\mathrm{C}$ is an ANR.

C.5.12. PROPOSITION. If $Y$ is an ANE for the class of all metrizable spaces with property $\mathrm{C}$, then $Y$ has the following property. If $A$ is a closed subset of $a$ 
metrizable space $X$ and $A$ has property $\mathrm{C}$, then any map $\phi: A \rightarrow Y$ extends to a map $\psi: N \rightarrow Y$ where $N$ is a neighborhood of $A$ in $X$.

The proof of Proposition 5.12 can be transformed into a proof of this proposition via the following remark. Lemma 5.11 provides a map $g: X \rightarrow Z$ from $X$ to a metrizable space $Z$ such that $g \mid A: A \rightarrow Z$ is a closed embedding and $Z-g(A)$ is countable dimensional. Hence $Z$ is the union of two subspaces which have property C: $g(A)$ and $Z-g(A)$. It follows that $Z$ has property C. (Indeed, it is easily proved that a metrizable space has property $\mathrm{C}$ if it is the union of countably many subspaces each having property C.) From this point, the proofs of 5.12 and C.5.12 coincide.

T. Dobrowolski has suggested generalizing Questions 5.13(a), (c) in the following way.

C.5.13. Questions. Consider the following four classes of metrizable spaces.

(a) Locally contractible spaces.

(b) ANE's for the class of all metrizable spaces with property $\mathrm{C}$.

(c) Metrizable spaces $Y$ with the following property. For every $y \in Y$ and every neighborhood $U$ of $y$ in $Y$, there is a neighborhood $V$ of $y$ in $Y$ such that if $A$ is a closed subset of a metrizable space $X$ and $A$ has property $\mathrm{C}$, then any map from $A$ to $V$ extends to a map from $X$ to $U$.

(d) Metrizable spaces $Y$ with the following property. For every $y \in Y$ and every neighborhood $U$ of $y$ in $Y$, there is a neighborhood $V$ of $y$ in $Y$ such that for each $n \geq 0$, each map from $S^{n}$ to $V$ extends to a map from $B^{n+1}$ to $U$.

Each of these classes of spaces is included in its successor. Class (a) is included in class (b) by Corollary C.5.10. A demonstration that class (b) is contained in class (c) is obtained by imitating the proof that (b) implies (c) in Theorem V.2.1, pp. 153-154 of $[\mathbf{H u}]$, with assistance from Proposition C.5.12. The inclusion of class (c) in class (d) is obvious. Is any of these inclusions proper?

\section{REFERENCES}

[AG] D. F. Addis and J. H. Gresham, A class of infinite dimensional spaces. Part I: Dimension theory and Alexandroff's problem, Fund. Math. 101 (1978), 195-205.

[A] F. D. Ancel, The locally flat approximation of cell-like embedding relations, Ph.D. Thesis, University of Wisconsin, Madison, 1976.

[AC] F. D. Ancel and J. W. Cannon, The locally flat approximation of cell-like embedding relations, Ann. of Math. (2) 109 (1979), 61-86.

[AP] S. Armentrout and T. M. Price, Decompositions into compact sets with UV properties, Trans. Amer. Math. Soc. 141 (1969), 433-442.

[C1] J. W. Cannon, Taming cell-like embedding relations, Geometric Topology (L. C. Glaser and T. B. Rushing, editors), Lecture Notes in Math., vol. 438, Springer-Verlag, New York, 1975, pp. 66-118.

[C2] - Taming codimension-one generalized submanifolds of $S^{n}$, Topology 16 (1977), 323-334.

[C3],$\Sigma^{2} H^{3}=S^{5} / G$, Rocky Mountain J. Math. 8 (1978), 527-532.

[C4] _ Shrinking cell-like decompositions of manifolds. Codimension three, Ann. of Math. (2) 110 (1979), 83-112.

[Ch] T. A. Chapman, Lectures on Hilbert cube manifolds, CBMS Regional Conf. Ser. in Math., no. 28, Amer. Math. Soc., Providence, R.I., 1976.

[DW] R. J. Daverman and J. J. Walsh, Examples of cell-like maps that are not shape equivalences (preprint).

[D] J. Dugundji, Topology, Allyn \& Bacon, Boston, Mass., 1966. 
[E] R. D. Edwards, The double suspension of certain homology 3-spheres is $S^{5}$, Notices Amer. Math. Soc. 22 (1975), A-334; Abstract \#757-G33.

[Ev] D. L. Everett, Embedding theorems for decomposition spaces, Houston J. Math. 3 (1977), 351-367.

[F] M. H. Freedman, The topology of four-dimensional manifolds, J. Differential Geom. 17 (1982), $357-453$.

[G] J. H. Gresham, A class of infinite-dimensional spaces. Part II: An extension theorem and the theory of retracts, Fund. Math. 106 (1980), 237-245.

[H1] W. E. Haver, Mappings between ANR's that are fine homotopy equivalences, Pacific J. Math. 58 (1975), 457-461.

[H2] _ A covering property for metric spaces, Topology Conf., Virginia Polytechnic Institute and State University (R. F. Dickman and P. Fletcher, editors), Lecture Notes in Math., vol. 375, Springer-Verlag, New York, 1974, pp. 108-113.

[H3 _ L L L Lally contractible spaces that are absolute neighborhood retracts, Proc. Amer. Math. Soc. 40 (1973), 280-284.

[H4] _ _ A near-selection theorem, General Topology Appl. 9 (1978), 117-124.

[Hu] S. T. Hu, Theory of retracts, Wayne State Univ. Press, Detroit, 1965.

[K1] G. Kozlowski, Factorization of certain maps up to homotopy, Proc. Amer. Math. Soc. 21 (1969), 88-92.

[K2] , Images of ANR's, Trans. Amer. Math. Soc. (to appear).

[Ku] K. Kuratowski, Topology. II, Academic Press, New York, 1968.

[L1] R. C. Lacher, Cell-like spaces, Proc. Amer. Math. Soc. 20 (1969), 598-602.

[L2] _ Cell-like mappings. I, Pacific J. Math. 30 (1969), 717-731.

[M] D. R. McMillan, Jr., A criterion for cellularity in a manifold, Ann. of Math. (2) 79 (1964), 327-337.

[N] J. Nagata, Modern dimension theory, Wiley, New York, 1965.

[P] R. Pol, A weakly infinite-dimensional compactum which is not countable dimensional, Proc. Amer. Math. Soc. 82 (1981), 634-636.

[Sm] S. Smale, A Vietoris mapping theorem for homotopy, Proc. Amer. Math. Soc. 8 (1957), 604-610.

[Sp] E. H. Spanier, Algebraic topology, McGraw-Hill, New York, 1966.

[SS] L. A. Steen and J. A. Seebach, Counterexamples in topology, 2nd ed., Springer-Verlag, New York, 1978.

[T] J. L. Taylor, A counterexample in shape theory, Bull. Amer. Math. Soc. 81 (1975), 629-632.

[To] H. Torunczyk, On CE-images of the Hilbert cube and characterization of Q-manifolds, Fund. Math. 106 (1981), 31-40.

[W] J. H. C. Whitehead, A certain open manifold whose group is unity, Quart. J. Math. 6 (1935), $268-279$.

Department of Mathematics, University of OKLAhoma, Norman, OKLAhoma 73019 